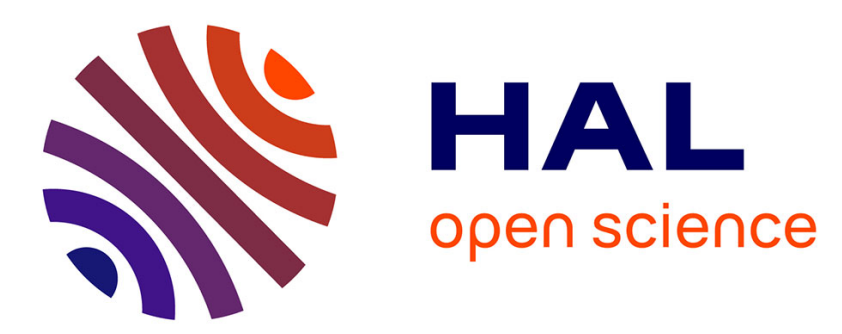

\title{
A high-order h-adaptive discontinuous Galerkin method for unstructured grids based on a posteriori error estimation
}

Francesca Basile, Jean-Baptiste Chapelier, Marta de La Llave Plata, Romain Laraufie, Pascal Frey

\section{To cite this version:}

Francesca Basile, Jean-Baptiste Chapelier, Marta de La Llave Plata, Romain Laraufie, Pascal Frey. A high-order h-adaptive discontinuous Galerkin method for unstructured grids based on a posteriori error estimation. AIAA Scitech 2021 Forum, Jan 2021, VIRTUAL EVENT, United States. 10.2514/6.20211696. hal-03380295

\section{HAL Id: hal-03380295 https://hal.science/hal-03380295}

Submitted on 15 Oct 2021

HAL is a multi-disciplinary open access archive for the deposit and dissemination of scientific research documents, whether they are published or not. The documents may come from teaching and research institutions in France or abroad, or from public or private research centers.
L'archive ouverte pluridisciplinaire HAL, est destinée au dépôt et à la diffusion de documents scientifiques de niveau recherche, publiés ou non, émanant des établissements d'enseignement et de recherche français ou étrangers, des laboratoires publics ou privés. 


\title{
A high-order $\boldsymbol{h}$-adaptive discontinuous Galerkin method for unstructured grids based on a posteriori error estimation
}

\author{
Francesca Basile* and Jean-Baptiste Chapelier ${ }^{\dagger}$ \\ ONERA - Université Paris-Saclay, Châtillon, 92322, France \\ Marta de la Llave Plata \\ ONERA - Université de Toulouse, Toulouse, 31055, France \\ Romain Laraufie ${ }^{\S}$ \\ Airbus Operations SAS, Toulouse, 31060, France \\ Pascal Frey ${ }^{\mathrm{I}}$ \\ Sorbonne Université, Paris, 75005, France
}

\begin{abstract}
In this paper, we present a mesh $\boldsymbol{h}$-adaptation strategy suited for the discontinuous Galerkin formulation of the compressible Navier-Stokes equations on unstructured grids, based on the simplicial remeshing library MMG. A novel a posteriori error estimator, combining the measure of the energy associated with the highest-order modes and the inter-element jumps, is used to build the metric field. The performance of the developed mesh adaptation algorithm is assessed for steady laminar viscous flows past a square cylinder and past a NACA0012 airfoil, and for the unsteady laminar viscous flow past a circular cylinder. The gain in accuracy for a given number of degrees of freedom (DoFs) is demonstrated for $p=1, p=2$ and $p=3$ polynomial degrees, with respect to uniformly refined simulations.
\end{abstract}

\section{Introduction}

Discontinuous Galerkin (DG) methods [1,2] are high-order finite element discretizations which were introduced in the early 1970's for the numerical simulation of the hyperbolic neutron transport equations [3]. These methods are based on the variational formulation of the governing equations and combine features of Finite Volume (FV) and Finite Element (FE) methods. In recent years, these methods have become very popular for the solution of nonlinear convection dominated flow problems [4]. Many aspects make DG methods particularly attractive, such as their high-order of accuracy achieved on arbitrary unstructured meshes, accurate description of curved boundaries and suitability to parallel computing thanks to a compact stencil. Another advantage of DG methods is the possibility to exploit $a$ posteriori jump and spectral error indicators for mesh adaptation. These error indicators are convenient thanks to their efficiency, locality, simplicity and low computational cost [5-8].

In particular the research community has begun to focus on $h p$-adaptive methods in which, one not only locally adapts the size $h$ of the mesh, but also the degree of the polynomials, $p$, within the element. An attractive property of $h p$-adaptivity lies in its exponential error convergence rate in terms of degrees of freedom [1], as opposed to a polynomial rate for fixed $p$ in the case of uniform $h$-refinement. The complication here is that the local error estimator is no longer sufficient to guide the adaptivity, because it indicates which element should be refined, but does not indicate whether it is better to refine the element by $h$ or $p$. A method for making that choice is called an $h p$-decision strategy. In practice $h p$-adaptive methods lead to the concentration of the degrees of freedom (DoFs) in regions of interest of the flow, in order to optimize both the computational cost and the accuracy of the simulations [9. 11$]$.

As regards $h$-adaptation, mainly two strategies can be used:

- element subdivision supporting nonconforming and hanging nodes, that can be isotropic [12-14] or anisotropic [15]-17], and mainly conformal for tetrahedral meshes [18,-20].

\footnotetext{
*Ph.D Student, ONERA, Departement of Aerodynamics, Aeroelasticity \& Acoustics, Airbus, Sorbonne Université, francesca.basile-ext@onera.fr

${ }^{\dagger}$ Research Engineer, ONERA, Departement of Aerodynamics, Aeroelasticity \& Acoustics, jean-baptiste.chapelier@onera.fr

$\doteqdot$ Senior Research Engineer, ONERA, Multi-physics for energetics department, marta.de_la_llave_plata@onera.fr

${ }^{\S}$ Engineer, Airbus, Flight Physics Capabilities, romain.laraufie@airbus.com

IProfessor of Mathematics, Sorbonne Université, Laboratoire Jacques Louis Lions, pascal.frey@ sorbonne-universite.fr
} 
- global remeshing, in which a new mesh is generated for the entire computational domain. The original mesh is then used to store the characteristics of the new mesh during regeneration. This new mesh is described using a Riemannian metric, based on the assumption that for an optimal mesh all edges lengths will have unit measure in the metric space. Both anisotropic [21,-24] and isotropic [25, 26] variants are found in the literature regarding this approach.

Different criteria, which dictate the modifications to be applied to the mesh, can be found in the literature, see [27] for a thorough review. One of common strategies, typically used in FE/FV contexts, consists in computing a metric from the Hessian matrix of the solution [24, 28-30]. Information from the Hessian has also been used by Remacle et al. in [31] in the context of DG based on a second order discretization $(p=1)$ to prescribe an anisotropy to the element and in [14] to compute the direction of shocks and other discontinuities for higher order DG discretizations. The definition of a metric tensor is difficult for high-order methods as the standard Hessian matrix approach assumes a linear approximation of the scalar quantity.

The extension of the Hessian approach to high-order methods has been performed for 2D metric-based mesh adaptation, based on minimizing the interpolation error of the solution by aligning the dominant principal direction of the Riemannian metric tensor with the direction of the maximum $(p+1)-t h$ derivative of the solution [32-34]. In the framework of high-order finite elements, the extension to very high-order solutions in 3D has been treated in [35] as a strongly non-linear optimization problem which needs the solution of an adjoint problem. The 3D metric-based anisotropic mesh adaptation for high-order is thus an open research topic. A simple approach not involving expensive complex optimization problems, which are generally not suitable for unsteady configurations, seems still out of reach in an industrial context. In addition, the solution of the adjoint problem requires the backward integration in time for unsteady problems, thus requiring the solution to be known at each previous time step.

Isotropic global-remeshing approaches, more easily adaptable to 3D, are also featured in the literature. Bernard et al. [36] solve two saddle point optimization problems that can be computed in a closed form for constant polynomial degree: one of them minimizes the global error in the domain while keeping constant the number of elements. The second problem allows for minimizing the number of elements while keeping the global constant in the domain. This algorithm does not perform node repositioning but only edge swaps, edge collapses and edge splits. Daviller et al. [25] use a sensor on the dissipation of kinetic energy in the context of LES simulations, averaged during the simulation and provided as field function to the remesher. Park et al. [26] propose a $h$-refinement of the mesh by inserting new nodes at the midpoint of the flagged edges (with the marking strategy driven by a quantity of interest) and reconnecting these nodes into new tetrahedra and boundary faces. Then they employ face and edge swapping to improve the mesh quality. In the context of isotropic $h$-adaptation Balan et al. [33] developed a fixed fraction marking strategy, where the marked elements are subdivided to form child elements.

Among the available mesh adaptation softwares using a metric-based strategy, based on the Delaunay method [37-43], MMG is a remeshing library developed by Bordeaux INP, CNRS, Sorbonne Université and INRIA [22, 44]. The MMG software, supporting both 2D and 3D remeshing (MMG2D and MMG3D) has been chosen in this work, relying on primitive operators like edge splitting, edge collapsing, edge swapping, and node relocation. The MMG library has been already used extensively in the context of FV and FE methods [25, 45-47].

The use of a metric-based approach shows promising results in the literature, is extensively used, and a number of global remeshing software are available, often under open-source licensing. Moreover, the metric-based remeshing strategy allows us to circumvent the complications associated with hanging nodes required by an element splitting-based strategy, which is not available in all flow solvers, and shows more flexibility in terms of prescribing a given size to an element. Indeed with a metric-based approach one can prescribe a precise size to an element, without being constrained by the arbitrary size induced by the splitting of previous elements.

Our work is motivated by the research of a simple, easily implementable mesh adaptation algorithm, which is expected to be rapidly converging towards the optimal mesh. The focus is on flow configurations relevant in an industrial context, for which a minimal number of adaptation steps is sought due to the size and complexity of the problems and the necessity of optimizing the simulation costs. In particular, the final envisaged applications involve hybrid RANS-LES flow simulations, featuring 3D configurations with a high number of degrees of freedom. The possibility to extend the algorithm to $3 \mathrm{D}$ in a straightforward manner is, in this context, an essential requirement. This $h$-adaptation procedure is intended to be extended to $h p$-adaptation with the introduction of an $h p$-decision strategy.

The present work focuses on isotropic mesh refinement strategies, considering the target applications of the present study. They include LES simulations of turbulent jets, and given the fundamental isotropic character of free-shear, small-scale turbulence [48], it can be expected that the ideal fully adapted mesh would be isotropic for this kind of physical phenomenon. The extension to anisotropic mesh adaptation will be addressed in future research. 
An inexpensive a posteriori error estimator which employs the solution itself to derive estimates of the discretization error, is used to control the size of the elements of the mesh. The error estimator couples two indicators, based on the measure of the energy contained in the highest-order modes of the modal expansion and on the element interface momentum jumps respectively. These sensors are coupled to guarantee a reasonable behaviour both for high- and low-degree polynomial approximations. The prescribed sizes are provided to the MMG2D remeshing library as an input size map, which outputs the newly adapted mesh. The solution on the previous mesh is projected on the newly adapted mesh. The gain in terms of number of degrees of freedom of the adaptation algorithm with respect to uniformly refined meshes, is evaluated by computing laminar flow simulations featuring polynomial degrees $p=1,2$ and 3 , in particular of the flow past a square cylinder at low Reynolds $R e=40$, past a NACA0012 airfoil at $R e=5000$ and Mach $M=0.5$ and past a circular cylinder at Reynolds $R e=100$.

This adaptation procedure is applied in the context of the unstructured compressible flow solver CODA, developed in partnership by Airbus, ONERA and DLR [49] and targets research and industrial aerodynamic problems of interest. This new CFD platform is designed for efficient parallel and heterogeneous architectures, applying modern software techniques to a wide range of multidisciplinary applications. The object-oriented CODA framework permits the integration of advanced inter-operable CFD components, including the implementation of two different flow solvers based on Finite Volume and Discontinuous Galerkin schemes, applied to the Navier-Stokes and the RANS equations.

The paper is organized as follows. Section [I] presents the details of the discretization of the Navier-Stokes equations using the Discontinuous Galerkin method, Section [III provides a thorough description of the $h$-adaptive procedure and Section $[\mathrm{IV}$ presents the application and assessment of the implemented $h$-adaptation algorithm to two steady configurations, the viscous laminar flows past a square cylinder at Reynolds 40 and past a NACA 0012 at Reynolds 5000, Mach 0.5 and $\alpha=1^{\circ}$, and to an unsteady configuration of the laminar flow past a circular cylinder at Reynolds 100. Concluding remarks and directions for future research are discussed in section $\mathrm{V}$.

\section{Discontinuous Galerkin discretization of the compressible Navier-Stokes equations}

This section describes the model equations for solving compressible fluid flow problems as well as the Discontinuous Galerkin discretization implemented in the flow solver CODA used in the present work.

\section{A. The compressible Navier-Stokes equations}

The motion of a compressible fluid in a three-dimensional domain $\Omega \in \mathbb{R}^{3}$ is described by the compressible Navier-Stokes (NS) equations, which take the form

$$
\begin{array}{cl}
\partial_{t} \mathbf{u}+\nabla \cdot\left(\mathbf{F}_{c}(\mathbf{u})-\mathbf{F}_{v}(\mathbf{u}, \nabla \mathbf{u})\right)=0, & \forall \mathbf{x} \in \Omega, t>0 \\
\mathbf{u}(\mathbf{x})=\mathbf{u}_{0}, & \forall \mathbf{x} \in \Omega
\end{array}
$$

with appropriate boundary conditions prescribed on $\partial \Omega$. The vector $\mathbf{u}$ represents the conservative variables, with $\rho$ being the density, $\mathbf{v}$ being the velocity vector and $E=\frac{p}{(\gamma-1) \rho}+\frac{\mathbf{v} \cdot \mathbf{v}}{2}$ the specific total energy. $p$ is the static pressure, $\gamma=\frac{C_{p}}{C_{v}}$ is the ratio of specific heats. $\mathbf{F}_{c}(\mathbf{u})$ and $\mathbf{F}_{v}(\mathbf{u}, \nabla \mathbf{u})$ are the convective and diffusive fluxes defined, respectively, as:

$$
\mathbf{F}_{c}(\mathbf{u})=\left[\begin{array}{c}
\rho \mathbf{v}^{T} \\
\rho \mathbf{v} \otimes \mathbf{v}+p \mathbf{I} \\
\rho E \mathbf{v}^{T}+p \mathbf{v}^{T}
\end{array}\right] \text { and } \mathbf{F}_{v}(\mathbf{u}, \nabla \mathbf{u})=\left[\begin{array}{c}
0 \\
\tau \\
\tau \cdot \mathbf{v}-\mathbf{q}^{T}
\end{array}\right]
$$

with:

$$
\begin{gathered}
\boldsymbol{\tau}=2 \mu \mathbf{S}^{D}=\mu\left(\boldsymbol{\nabla} \mathbf{v}+(\boldsymbol{\nabla} \mathbf{v})^{T}-\frac{2}{3} \boldsymbol{\nabla} \cdot \mathbf{v I}\right) \\
\mathbf{q}=-\lambda \boldsymbol{\nabla} T \\
\mathrm{p}=\rho R T
\end{gathered}
$$


where $\mu$ is the dynamic viscosity, $\mathbf{S}^{D}$ is the deviatoric component of the strain-rate tensor $\mathbf{S}=\frac{1}{2}\left(\boldsymbol{\nabla} \mathbf{v}+(\boldsymbol{\nabla} \mathbf{v})^{T}\right), T$ is the temperature, $R$ is the specific gas constant, $\lambda=\mu \frac{C_{p}}{P r}$ is the thermal conductivity, with $\operatorname{Pr}$ the Prandtl number. In this work, the value $\operatorname{Pr}=0.72$ is used.

\section{B. DG Discretization}

The DG discretization used in this work is based on a modal approach that relies on the use of a hierarchical and orthogonal polynomial basis for the Galerkin projection. Numerical integration is efficiently performed by means of a Gaussian quadrature. In this work steady solutions are obtained by employing an implicit backward-Euler scheme relying on the matrix-free GMRES block-Jacobi method with LU-Jacobi as preconditioner. The explicit time integration for the last unsteady test case is performed by using a classical fourth-order Runge-Kutta scheme [50]. The DG method implemented in the CODA solver is briefly outlined below.

We start by defining a shape-regular partition of the domain $\Omega$, into $N$ non-overlapping and non-empty cells $K$ of characteristic size $h$. We also define the sets $\mathcal{E}_{i}$ and $\mathcal{E}_{b}$ of interior and boundary faces in $\Omega_{h}$, such that $\mathcal{E}_{h}=\mathcal{E}_{i} \cup \mathcal{E}_{b}$.

Let $\mathcal{V}_{h}^{p}=\left\{\phi_{h} \in L^{2}\left(\Omega_{h}\right):\left.\phi\right|_{\mathcal{K}} \in \mathcal{P}^{p}(\mathcal{K}), \forall \mathcal{K} \in \Omega_{h}\right\}$ be the functional space formed by piece-wise polynomials of degree at most $p$, and $\left(\phi_{\mathcal{K}}^{1}, \ldots, \phi_{\mathcal{K}}^{N_{p}}\right) \in \mathcal{P}^{p}(\mathcal{K})$ a hierarchical and orthonormal basis of $\mathcal{V}_{h}^{p}$, of dimension $N_{p}$, confined to $\mathcal{K}$. The solution in each element is thus expressed as

$$
\mathbf{u}_{h}(\mathbf{x}, t)=\sum_{l=1}^{N_{p}} \phi_{\mathcal{K}}^{l}(\mathbf{x}) \mathbf{u}_{\mathcal{K}}^{l}(t), \forall \mathbf{x} \in \mathcal{K}, \quad \mathcal{K} \in \Omega_{h}, \forall t \geq 0
$$

The polynomial coefficients $\left(\mathbf{u}_{\mathcal{K}}^{l}\right)_{1 \leq l \leq N_{p}}$ represent the degrees of freedom of the discrete problem in element $\mathcal{K}$. The shape functions are polynomials that can be chosen arbitrarily. A methodology developed by Bassi et al. [51] consists in defining a starting set of monomial basis functions in each (arbitrarily shaped) element and applying a modified Gram-Schmidt orthonormalization procedure. The resulting basis yields a diagonal mass matrix in each element of the discretization, simplifying the resolution of the variational formulation. The conservation law is discretized in physical space by using a discontinuous Galerkin method and the semi-discrete variational form of the system of equations (11) thus reads: find $\mathbf{u}_{h}$ in $\mathcal{V}_{h}^{p}$ such that $\forall \phi_{h} \in \mathcal{V}_{h}^{p}$ we have

$$
\int_{\Omega_{h}} \phi_{h} \partial_{t} \mathbf{u}_{h} d V+\mathcal{L}_{c}\left(\mathbf{u}_{h}, \phi_{h}\right)+\mathcal{L}_{v}\left(\mathbf{u}_{h}, \phi_{h}\right)=0
$$

In eq. (7) $\mathcal{L}_{c}$ and $\mathcal{L}_{v}$ represent the weak form of the convective and viscous terms respectively. The following notations are introduced: for a given interface $e$ in $\mathcal{E}_{i}$ we define the average operator as $\{\{\mathbf{u}\}\}=\left(\mathbf{u}^{+}+\mathbf{u}^{-}\right) / 2$, the jump operator is defined as $[[\mathbf{u}]]=\mathbf{u}^{+} \otimes \mathbf{n}-\mathbf{u}^{-} \otimes \mathbf{n}$ where $\mathbf{u}^{+}$and $\mathbf{u}^{-}$are the traces of the variable $\mathbf{u}$ at the interface between elements $\mathcal{K}^{+}$and $\mathcal{K}^{-}$. The DG discretization of the convective terms then reads

$$
\mathcal{L}_{c}\left(\mathbf{u}_{h}, \phi_{h}\right)=-\int_{\Omega_{h}} \mathbf{F}_{c}\left(\mathbf{u}_{h}\right) \cdot \nabla_{h} \phi_{h} d V+\int_{\mathcal{E}_{i}}\left[\left[\phi_{h} \rrbracket \mathbf{h}_{c}\left(\mathbf{u}_{h}^{+}, \mathbf{u}_{h}^{-}, \mathbf{n}\right) d S+\int_{\mathcal{E}_{b}} \phi_{h}^{+} \mathbf{F}_{c}\left(\mathbf{u}_{b}\right) \cdot \mathbf{n} d S\right.\right.
$$

where the boundary values $\mathbf{u}_{b}=\mathbf{u}_{b}\left(\mathbf{u}_{h}^{+}, \mathbf{u}_{e x t}, \mathbf{n}\right)$, with $\mathbf{u}_{\text {ext }}$ a reference external state computed so that the boundary conditions are satisfied on $\mathcal{E}_{b}$. The numerical flux $\mathbf{h}_{c}$ is chosen such that it is consistent and compact. In this work we use the Local Lax Friedrichs (LLF) flux [52] for steady simulations and the Roe flux [53] with an entropy fix similar to that of Harten [54] for unsteady simulations.

The discretization of the viscous terms is this work is performed using the BR1 [55] and the BR2 [56, 57] approaches of Bassi and Rebay which are implemented in CODA. These approaches rely on the definition of the conservative variables gradients as auxiliary variables $\sigma=\nabla \mathbf{u}$ which verify the following equations:

$$
\begin{aligned}
\boldsymbol{\sigma}-\nabla \mathbf{u} & =0 \\
\partial_{t} \mathbf{u}+\nabla \cdot \mathbf{F}_{c}(\mathbf{u})+\nabla \cdot \mathbf{F}_{v}(\mathbf{u}, \boldsymbol{\sigma}) & =0
\end{aligned}
$$

This leads to the introduction of the so called global lifting operator $\mathbf{L}_{h}$ such that:

$$
\sigma_{h}=\nabla_{h} \mathbf{u}_{h}+\mathbf{L}_{h}
$$


and $\mathbf{L}_{h}$ satisfies the following condition

$$
\int_{\Omega_{h}} \phi_{h} \mathbf{L}_{h} d V=-\int_{\mathcal{E}_{i}}\{\{\phi\}\}\left[\left[\mathbf{u}_{u}\right] d S-\int_{\mathcal{E}_{b}} \frac{\phi^{+}}{2}\left(\mathbf{u}_{h}^{+}-\mathbf{u}_{b}\right) \otimes \mathbf{n} d S\right.
$$

The discrete variational form of the viscous term for the BR1 method therefore takes the form

$$
\begin{aligned}
\mathcal{L}_{v}\left(\mathbf{u}_{h}, \phi_{h}\right)= & -\int_{\Omega_{h}} \mathbf{F}_{v}\left(\mathbf{u}_{h}, \nabla_{h} \mathbf{u}_{h}+\mathbf{L}_{h}\right) \cdot \nabla_{h} \phi_{h} d V-\int_{\mathcal{E}_{i}}\left[\left[\phi_{h}\right]\left\{\left\{\mathbf{F}_{v}\left(\mathbf{u}_{h}, \nabla_{h} \mathbf{u}_{h}+\mathbf{L}_{h}\right)\right\}\right\} \cdot \mathbf{n} d S\right. \\
& -\int_{\mathcal{E}_{b}} \phi_{h}^{+} \mathbf{F}_{v}\left(\mathbf{u}_{b}, \nabla \mathbf{u}_{b}+\mathbf{L}_{h}\right) \cdot \mathbf{n} d S
\end{aligned}
$$

In the BR2 method, Bassi and Rebay proposed to overcome some drawbacks of this method due to its marginal stability and non compactness by introducing in the formulation the local lifting operators $\mathbf{l}_{h}^{e}: \mathbf{L}_{h}=\sum_{e \in \partial \Omega_{h}} \mathbf{l}_{h}^{e}$ where $\mathbf{l}_{h}^{e}$ has support on the elements adjacent to $e \in \mathcal{E}_{i}$ and is obtained from the solution of the following problem on internal faces:

$$
\int_{\mathcal{K}^{+} \cup \mathcal{K}^{-}} \phi_{h} \mathbf{l}_{h}^{e} d V=\int_{\partial \mathcal{K}^{+} \cup \partial \mathcal{K}^{-}}\left\{\left\{\phi_{h}\right\}\right\}\left[\left[\mathbf{u}_{h}\right]\right] d S
$$

The viscous discretization for the BR2 method becomes here

$$
\begin{aligned}
\mathcal{L}_{v}\left(\mathbf{u}_{h}, \phi_{h}\right)= & -\int_{\Omega_{h}} \mathbf{F}_{v}\left(\mathbf{u}_{h}, \nabla_{h} \mathbf{u}_{h}+\mathbf{L}_{h}\right) \cdot \nabla_{h} \phi_{h} d V-\int_{\mathcal{E}_{i}}\left[\left[\phi_{h}\right]\left\{\left\{\mathbf{F}_{v}\left(\mathbf{u}_{h}, \nabla_{h} \mathbf{u}_{h}+\eta \mathbf{l}_{h}^{e}\right)\right\}\right\} \cdot \mathbf{n} d S\right. \\
& \left.-\int_{\mathcal{E}_{b}} \phi_{h}^{+} \mathbf{F}_{v}\left(\mathbf{u}_{b}, \nabla \mathbf{u}_{b}+\eta \mathbf{l}_{h}^{e}\right) \cdot \mathbf{n} d S\right)
\end{aligned}
$$

where $\eta$ is a numerical parameter that ensures the stability of the method, set to 1 in this work.

\section{III. $h$-adaptation algorithm}

In the present section, we describe in detail the $h$-adaptation strategy used in this research. It is based on the coupling of accurate DG-based indicators with the external remeshing library suited for unstructured simplicial meshes MMG.

The adaptation procedure is driven by a novel error estimator inspired by the work of Colombo et al. [58], which combines a measure of the energy associated with the highest-order polynomial modes [59] and the inter-element jumps [36]. This new estimator controls the solution accuracy within the domain, identifying the regions lacking/exceeding the requested resolution. In this work these regions will be refined/coarsened by decreasing/increasing the size of the element.

\section{A. Error estimator}

In the literature we can find refinement indicators based on a combination of two different types of error estimators. In [9], for example Dolejší et al. combine a residual-based and a non-conformity error estimator. In [58] Colombo et al. propose to combine an indicator based on the magnitude of the solution jumps at grid cell interfaces and one which involves the decay rate of the modal coefficients.

In this work we combine two error estimators in the same spirit as Colombo et al. in [58] who observed by numerical experiments that an error estimator based on the jumps at the interfaces of the elements is reliable for any polynomial degree, whereas an error indicator based on the high-order modes (hereafter termed SD for spectral decay) is reliable only for $p>1$. The SD estimator is accurate but can yield strong cell-to-cell variations, hence the interest of coupling such indicator with a jump error estimator is that the latter identifies a larger region for adaptation and smooths out the overall indicator map. According to these observations, they implemented a combination of the two indicators, after normalization over the minimum and maximum values.

In this work, we follow the same idea by combining two error estimators, one based on the energy of the highest-order modes, $\epsilon_{\mathrm{SSED}}$ and the other one based on the jumps across element interfaces, $\epsilon_{\mathrm{JUMP}}$.

The first error estimator is the Small Scale Energy Density (SSED) [12, 59], which is based on a measure of the error $\mathbf{E}_{\mathcal{K}}$ of the numerical solution $\mathbf{u}$ with respect to the exact solution $\mathbf{u}_{e x}$ in the element by computing the norm of the 
difference between the numerical solution $\mathbf{u}_{h, p}$ and the projection of the numerical solution on the reduced-order space $\mathcal{V}_{h}^{p-1}$, namely $\mathbf{u}_{h, p-1}$ :

$$
\mathbf{E}_{\mathcal{K}}=\left\|\mathbf{u}_{h}-\mathbf{u}_{e x}\right\| \simeq\left\|\mathbf{u}_{h, p}-\mathbf{u}_{h, p-1}\right\|
$$

The error based on the norm of the high-order modes of the momentum vector is normalized by the volume of the element (as done by Naddei et al. in [59]). This modification been found to provide better results compared to the classical SSED indicator not normalized by the volume proposed in [12] when a mesh with large variation in element size in the domain is employed. The final formulation reads:

$$
\epsilon_{\mathrm{SSED}, \mathcal{K}}^{2}=\frac{\int_{\mathcal{K}}\left\|(\rho \mathbf{v})_{h, p}-(\rho \mathbf{v})_{h, p-1}\right\|^{2} d V}{|\mathcal{K}|}=\frac{\left\|(\rho \mathbf{v})_{h, p}-(\rho \mathbf{v})_{h, p-1}\right\| \|_{L^{2}(\mathcal{K})}^{2}}{|\mathcal{K}|}
$$

where $(\rho \mathbf{v})_{h}$ is the momentum vector and $|\mathcal{K}|$ is the volume of the element.

The second error estimator is similar to the one found in [36]. The average value of the conservative variables at a given interface $e$ in $\mathcal{E}_{i}$ is used to approximate the exact solution $\mathbf{u}_{e x}^{+}$:

$$
\mathbf{u}_{e x}^{+} \simeq \frac{1}{2}\left(\mathbf{u}_{h}^{+}+\mathbf{u}_{h}^{-}\right)
$$

where $\mathbf{u}_{h}^{+}$and $\mathbf{u}_{h}^{-}$are respectively the traces of the variable $\mathbf{u}$ at the interface between elements $\mathcal{K}^{+}$and $\mathcal{K}^{-}$. We then define the error $\mathbf{E}_{e}$ on the interface $e$ as half the jump of the variable traces across the interfaces:

$$
\mathbf{E}_{e}=\left\|\mathbf{u}_{h}^{+}-\mathbf{u}_{e x}^{+}\right\| \simeq \frac{1}{2}\left\|\mathbf{u}_{h}^{+}-\mathbf{u}_{h}^{-}\right\|
$$

and we consider an averaged error over each interface $e$, for the norm of the momentum vector

$$
\epsilon_{\mathrm{JUMP}, e}^{2}=\frac{\int_{\partial K_{e}}\left\|(\rho \mathbf{v})_{h}^{+}-(\rho \mathbf{v})_{h}^{-}\right\|^{2} d S}{4\left|\partial \mathcal{K}_{e}\right|}=\frac{\left\|(\rho \mathbf{v})_{h}^{+}-(\rho \mathbf{v})_{h}^{-}\right\|_{L^{2}\left(\partial \mathcal{K}_{e}\right)}^{2}}{4\left|\partial \mathcal{K}_{e}\right|}
$$

For consistency with the dimensions of the SSED indicator in equation (19), the error indicator here is normalized by the area of each interface $\left|\partial \mathcal{K}_{e}\right|$ (or for 2D problems the length of the edge $e$ ). For each element $\mathcal{K}$ we compute the error estimator by the following simple rule:

$$
\epsilon_{\mathrm{JUMP}, \mathcal{K}}^{2}=\frac{1}{3} \sum_{e=1}^{3} \epsilon_{\mathrm{JUMP}, e}^{2}
$$

Both indicators are normalized by their respective maximum and minimum values over the whole domain (min-max normalization) before the coupling:

$$
\epsilon_{\mathcal{K}}=\operatorname{INT}\left(\frac{p}{\max (2, p)}\right) \epsilon_{\mathrm{SSED}, \mathcal{K}}+\frac{1}{\max (1, p)} \epsilon_{\mathrm{JUMP}, \mathcal{K}}
$$

where $p$ is the polynomial degree of the numerical solution and INT represents an integer division (meaning that $\epsilon_{\mathrm{SSED}, \mathcal{K}}$ is set to 0 for $\left.p \in[0,1]\right)$.

\section{B. Mesh metric prescription}

\section{Continuous mesh framework}

The mesh is modeled as a continuous medium: at the continuous level, we consider mesh elements being represented by ellipsoids (ellipses in 2D). In this geometric representation, the size of the element is its volume (area in 2D), the shape is associated with the ratio of the lengths of its semi-axes and the orientation is provided by its principal axis vectors [44]. Therefore the control of the element size can be achieved by specifying a metric tensor $\mathcal{M}(x)$ to prescribe the size, shape and orientation of mesh elements over the whole domain. In the $2 \mathrm{D}$ case, $\mathcal{M}(x)$ is a $2 \times 2$ symmetric positive definite matrix. In the continuous framework a metric tensor $\mathcal{M}$ is a continuous element [21]. A discrete 
element is unit with respect to a continuous element $\mathcal{M}$ if the length of all its edges is unit in the metric $\mathcal{M}$. We define the symmetric definite positive metric tensor as

$$
\mathcal{M}(\mathbf{x})=\mathcal{R} \Lambda \mathcal{R}^{T}
$$

where $\mathcal{R}$ is the square matrix whose $i$-th column is the eigenvector $\left(\mathbf{v}_{i}\right)_{i=1,2}$ of $\mathcal{M}$ and $\Lambda$ is the diagonal matrix whose diagonal elements are the corresponding eigenvalues $\Lambda_{i i}=\lambda_{i}$. The matrix $\Lambda$ thus prescribes the size and shape of the element, while the matrix $\mathcal{R}$ prescribes its orientation.
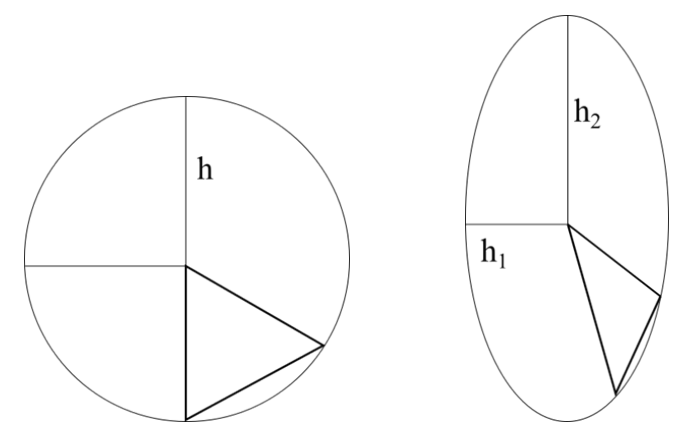

Fig. 1 Isotropic and anisotropic continuous elements, with associated unit elements.

The element sizes are then linked to the eigenvalues by $h_{1}=1 / \sqrt{\lambda_{1}}, h_{2}=1 / \sqrt{\lambda_{2}}$.

For an isotropic metric the semi-axes of the ellipse have the same length, and the two eigenvalues (and the two sizes) associated with the ellipse coincide, i.e. $h_{1}=h_{2}=h_{n}$, while the $i$-th eigenvectors are simply the $i$-th coordinate vectors.

$h_{n}$ denotes the characteristic size associated with a node $n$ from this point on.

In order to avoid confusion with subscripts and superscripts in the following, we note that the subscript $\mathcal{K}$ refers to the elements and the subscript $n$ refers to the mesh nodes, while the superscript ${ }^{*}$ represents the quantities required by the adaptation module in the current adaptation step.

\section{Mesh metric prescription principle}

At the end of the simulation, we compute the error estimator from the solution in each mesh element as $\epsilon_{\mathcal{K}}$. Then the updated element size $h_{\mathcal{K}}^{*}$ is imposed, for each mesh element $\mathcal{K}$, following the idea of Bernard et al.[36] based on the a priori estimation that for a DG method the $L^{2}$ norm of the error in the solution decays as $O\left(h^{p+1}\right)$ [2]:

$$
h_{\mathcal{K}}^{*}=h_{\mathcal{K}}\left(\frac{\epsilon_{\mathcal{K}}^{*}}{\epsilon_{\mathcal{K}}}\right)^{\frac{1}{p+1}}
$$

where $h_{\mathcal{K}}$ is the characteristic size of the element $\mathcal{K}, \epsilon_{\mathcal{K}}$ is the error estimator computed on the element $\mathcal{K}, \epsilon_{\mathcal{K}}^{*}$ is the globally imposed target error and $p$ is the degree of the polynomial approximation of the solution. Despite the fact that DG methods are element-based methods, where the characteristic size associated with the element $\mathcal{K}$ has a more natural definition, the input size-field that MMG requires is node-based. Therefore a volume-weighted average of the element-based values of the error estimator $\epsilon_{\mathcal{K}}$ is computed based on its values at the elements surrounding each node. This node-based error estimator will be denoted as $\epsilon_{n}$.

The formula in eq. 26) is then evaluated at the nodes, and the size $h_{n}^{*}$ is imposed at the node $n$ as

$$
h_{n}^{*}=h_{n}\left(\frac{\epsilon_{n}^{*}}{\epsilon_{n}}\right)^{\frac{1}{p+1}}
$$

where $h_{n}$ represents the characteristic size, i.e. the size of the semi-axis of the ellipse, associated with the node $n$. In an isotropic context $h_{n}$ measures the length of the edge of the equilateral triangles surrounding the node $n$. In practical terms MMG can provide as output of the remeshing process a non-scalar matrix, which means that it introduces some anisotropy. In this case the eigenvalues associated to the metric are not identical, and the characteristic length of the previous mesh is considered equal to $h_{n}=\left(h_{1} \cdot h_{2}\right)^{1 / 2}=\left(\lambda_{1} \cdot \lambda_{2}\right)^{-1 / 4}$, where $\lambda_{1}$ and $\lambda_{2}$ are the eigenvalues associated 
to the metric tensor output of MMG, obtained by eigen-decomposition. $\epsilon_{n}$ is the volume-weighted error estimator at the node $n$ and $\epsilon_{n}^{*}$ a target error estimator we impose to all the mesh nodes as described below.

Adopting this strategy, the mesh regions presenting a value of the error estimator lower than or equal to the target error are kept unchanged. On the other hand the regions with the highest values of error estimators are maximally refined (with respect to user-defined thresholds described in the following section). Finally the regions with the lowest values of the error estimators are maximally coarsened, and the regions with intermediate values are gradually refined/coarsened of a factor depending on the ratio between the target error and the value of the error estimator.

The input size-field is given for each node of the mesh in the form of a scalar matrix $\mathcal{M}^{*}=\Lambda^{*}=\operatorname{diag}\left(h_{n}^{*-2}, h_{n}^{*-2}\right)$ to the remesher MMG2D, allowing all primitive operators present in the remeshing library, i.e. edge splitting, edge collapsing, edge swapping, and node relocation. This step of the refinement process is seamless for the user.

The first step consists in obtaining from the initial mesh metric $h_{n}$ which is calculated by MMG2D from a preliminary remeshing step.

\section{User-defined parameters}

Two user-defined parameters, the maximum refinement factor $r_{h}$ and the maximum coarsening $c_{h}$ factor are needed to avoid uncontrolled or unwanted size changes between two subsequent adaptation steps. The values of $1 / r_{h}$ and $c_{h}$ are respectively the minimum and the maximum allowed ratios $h_{n}^{*} / h_{n}$. This means that for a given $r_{h}$ and $c_{h}$, we have $h_{n} / r_{h} \leq h_{n}^{*} \leq c_{h} h_{n}$, i.e. in one adaptation step the edge can increase its size by at most $c_{h}$ times the initial size, and decrease it by at most $r_{h}$ times.

The choice of the target error $\epsilon_{n}^{*}$ to prescribe to all mesh nodes is given by a revisited fix-fraction marking strategy, which allows for making the mesh adaptation algorithm to converge rapidly to the optimal mesh. The classical version of the fix-fraction marking strategy controlling the percentage of the mesh to refine and the percentage to coarsen showed poor results in this context. The prescribed decrease/increase of sizes here directly depends on the magnitude of the error estimator. A strong gap in the error estimator magnitude between the highest value and the mean value on the domain would lead to a localised refinement only in zones with highest intensity of the error estimator, and an insufficient refinement in zones with medium intensity of the estimator. This leads to an early and unwanted stop of the refinement algorithm.

The strategy we propose in this work allows for controlling the number of elements in the mesh, in order to prescribe the appropriate target error. This is made thanks to two approximations.

The first assumes that the number of nodes $N^{*}$ in the new mesh can be computed thanks to the prescribed reduction (and increment) of nodes sizes:

$$
N^{*}=\sum_{n}\left(\frac{h_{n}}{h_{n}^{*}}\right)^{d}
$$

$d$ is the number of dimensions of the mesh. The second one assumes that the ratio between the number of nodes and elements in the mesh is approximately constant for isotropic meshes.

The user-defined parameter $f_{C}$ used to define the target error thus corresponds to the factor of the mesh-nodes number increase at each adaptation step.

The target error is then defined with an iterative procedure, using the bisection method from the package python SciPy to solve the equation:

$$
N^{*}\left(\epsilon^{*}\right)-N_{C}=0
$$

where $N_{C}=f_{C} \cdot N$ is the number of nodes wanted in the following adaptation step.

Starting from the lowest error as a guess of the target error, the new lengths are computed with the equation (27), the new number of nodes is computed with the equation (28) and the iterative procedure results in the target error $\epsilon^{*}$ needed to obtain $N_{C}=f_{C} \cdot N$ nodes.

Once the mesh has been adapted, the solution from the previous mesh is projected to the adapted one by means of an $L^{2}$ projection.

\section{Summary of the required parameters and the $\boldsymbol{h}$-adaptive procedure}

The parameters needed to perform the adaptation are listed below, distinguishing between parameters needed by MMG and parameters needed by the external adaptation module coupled with MMG and CODA: 
- $h_{\text {grad }}$ : needed by MMG, the gradation value which controls the ratio between two adjacent edges (with a gradation of $h_{\text {grad }}$, two adjacent edges $h_{1}$ and $h_{2}$ must respect that $\frac{1}{h_{\text {grad }}} \leq \frac{h_{1}}{h_{2}} \leq h_{\text {grad }}$.

- $h_{\text {max }}$ : needed by MMG, the maximum allowed size of an edge $h_{n}$.

- $h_{\text {min }}$ : needed by MMG, the minimum allowed size of an edge $h_{n}$.

- $r_{h}$ : needed by the adaptation module, the maximum refinement factor, which controls the refinement between two subsequent adaptation steps.

- $c_{h}$ : needed by the adaptation module, the maximum coarsening factor, which controls the coarsening between two subsequent adaptation steps.

- $f_{C}$ : needed by the adaptation module, is used to define the target error $\epsilon^{*}$ of the current adaptation step as the error which would provide the increasing of $f_{C}$ times the total nodes of the mesh.

The $h$-adaptive procedure can be summarized, for a given step:

- The simulation is performed on the current mesh.

- The a posteriori error estimator $\epsilon$ is then computed on the current mesh.

- The target error $\epsilon^{*}$ is chosen in order to fulfill the given refinement criterion.

- The current isotropic metric, represented by the size of the edge $h_{n}$ is adapted to the new $h_{n}^{*}$ provided by the formulation (27).

- The new metric is given to MMG which performs the remeshing step.

- Once the mesh has been adapted, the solution on the previous mesh is projected on the adapted one.

\section{Extension to unsteady periodical flows}

The adaptation strategy can be applied similarly for static $h$-adaptation of unsteady periodical flows. In this case the adaptive algorithm is applied once the periodic state of the flow is reached. Instead of the instantaneous values, the time-average of the error estimator accumulated for a given period is considered.

\section{Results}

\section{A. Steady flows}

The choice of parameters of the adaptation process for the simulations of the square cylinder and NACA0012 airfoil using $p=1, p=2$ and $p=3$ polynomial degrees is as follows:

- $h_{\text {grad }}=1.4$, which is higher than the default value of MMG2D $h_{\text {grad }}=1.3$. This value shows a good compromise between excessive refinement induced by a low value of $h_{\text {grad }}$, and the loss of isotropy induced by a high values.

- $h_{\text {min }}$ is set to $10^{-4} L$ (with $L$ the length of the side of the cylinder), which proved to be a good compromise between excessive refinement due to a low $h_{\min }$ and degradation of the solution due to a high $h_{\min }$. In figure 5 and 3 , a sensitivity study on this parameter has been performed for the test case of the flow past a square cylinder.

- $h_{\max }$ is set as the length of the domain where far-field boundary conditions are imposed.

- $r_{h}=c_{h}=2$ such that between two steps of the adaptation process, the edge of the equilateral triangle can at most double or halve its size.

- $f_{C}=1.3$ is used for $p=1, p=2, p=3$ simulations. By using the same value for the three polynomial degrees we expect a reduced number adaptation steps for higher-order simulations because of their faster mesh convergence properties.

Initial meshes are easily created with MMG2D by providing a uniform coarse metric and forcing the location of the mesh nodes on the wall. By using the mesh smoothing capability of MMG2D with hgrad $=1.4$, the resulting mesh is uniform and very coarse far away from the body and presents a smooth refinement towards the solid boundaries.

Moreover the initial metric at the nodes needed by the adaptation module is provided directly by the remesher.

The error on drag and lift coefficients is computed as the absolute value of the difference between the coefficient of the adaptive simulation at the $i-t h$ step, $\operatorname{Coef}_{i}$, and the reference coefficient Coef $r e f$ extracted from the simulation with the finest resolution, normalized by the latter.

$$
\mid \Delta \text { Coef }|=| \frac{\text { Coef }_{i}-\text { Coef }_{\text {ref }}}{\text { Coef }_{\text {ref }}} \mid
$$

The error on the $h$-adapted meshes is compared to uniformly refined meshes, obtained by successive remeshing with 
MMG. The uniformly refined meshes are produced by prescribing at the nodes a size which is half the size of the previously refined mesh. Basically we mark for maximum refinement all nodes and use the same remeshing algorithm used for $h$-adaptation.

\section{Laminar steady flow past a square cylinder at Reynolds $=40$}

The adaptation algorithm is tested on the laminar steady flow past a square cylinder at Reynolds $R e=40$ and $M a c h=0.1$ at zero incidence. The square cylinder test case is a well-known case in the literature, studied among others by Sen et al. in [60] and already used for validation of mesh adaptation algorithms both in the steady and unsteady configurations, by Li et al. [61], Chalmers in [62] and Hoffman et al. in [63].

The initial mesh consists in 312 elements and is used for $p=1, p=2$ and $p=3$. The domain is a square of side length $2000 L$ where $L$ is the side length of the square cylinder, which is centered at the position $(0,0)$. Free-stream far-field non-reflecting boundary conditions have been imposed on the external boundaries.

The error on the drag coefficient integrated over the walls of the square cylinder $C_{D}$ is evaluated for the different meshes by computing the difference between the values obtained on the adapted mesh and those from a reference $p=3$ simulation. The reference consists in the final adapted simulation on a 29047 elements mesh yielding a total of $3 \times 10^{5}$ DoFs. The reference adaptation starts from an initial mesh of higher quality than the one used in the present work. The choice of using an adapted simulation as reference is driven by the presence of the geometrical singularities. The convergence rate of the error obtained by the uniform $h$-refinement is not the theoretical one, i.e. the error as a function of the square root of the number of degrees of freedom does not decrease as $O\left(h^{p+1}\right)$. Generating the reference solution using uniform mesh refinement in this case would require a prohibitive number of degrees of freedom. The $C_{D}$ and the length of the recirculation bubble are compared with those found in the literature in table 1 , showing good accordance.

\begin{tabular}{l|l|l|l|l}
\hline & \multicolumn{1}{|c|}{$C_{D}$} & $C_{\text {Dpres }}$ & $C_{\text {Dfric }}$ & \multicolumn{1}{c}{$L / D$} \\
\hline Sen et al. [60] & 1.6680 & - & - & 2.8552 \\
\hline Meliga et al. [64] & 1.67 & - & - & - \\
\hline current & 1.643049 & 1.417378 & 0.225671 & 2.8318
\end{tabular}

Table 1 Steady square cylinder flow at $R e=40$. Integral flow quantities in the literature and for the present reference.
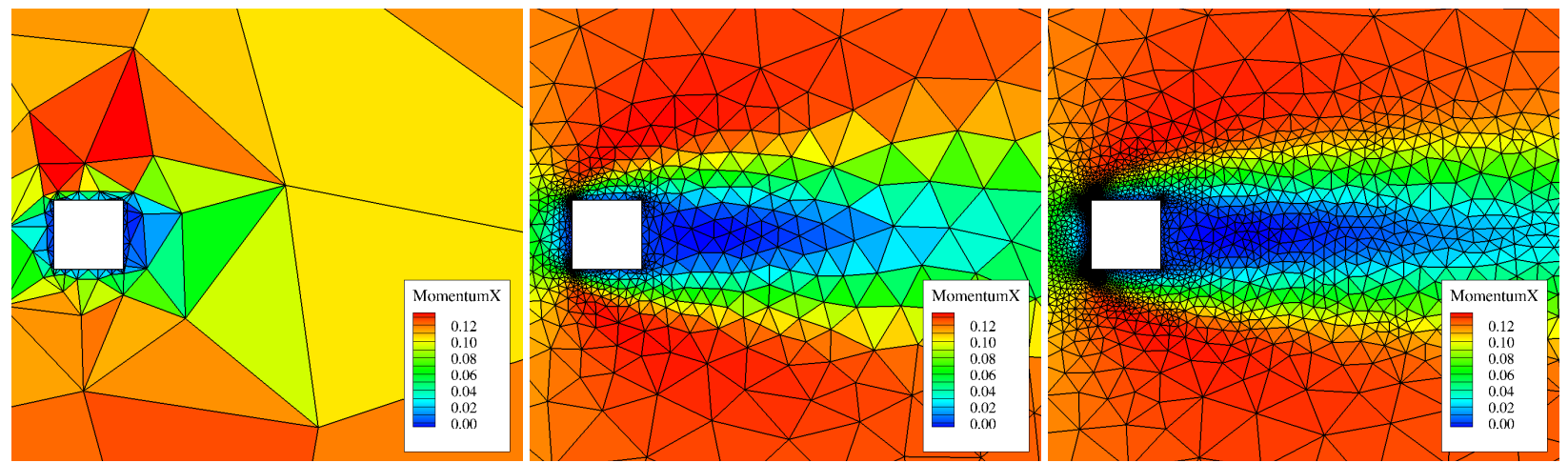

Fig. 2 Steady square cylinder flow at $R e=40$. Zoom of the x-momentum iso-contours of the cell-averaged solution on the initial mesh ( 312 elements), on the $3^{\text {rd }}$ adapted mesh (2087 elements) and on the $9^{\text {th }}$ adapted mesh ( 8890 elements), for the $p=1$ simulations.

Several adaptation steps have been performed with the three spatial discretizations. We show in figure 2 the solution of the Mach number on the initial mesh, after 3 adaptation steps and after 9 steps, for the $p=1$ simulations. The geometric singularities are massively refined in first place, especially the front corners, then the shear layers around the body and the wake are progressively refined over the course of the mesh adaptation process.

The error versus the number of degrees of freedom is shown for the performed simulations in figure 3 . Respectively 

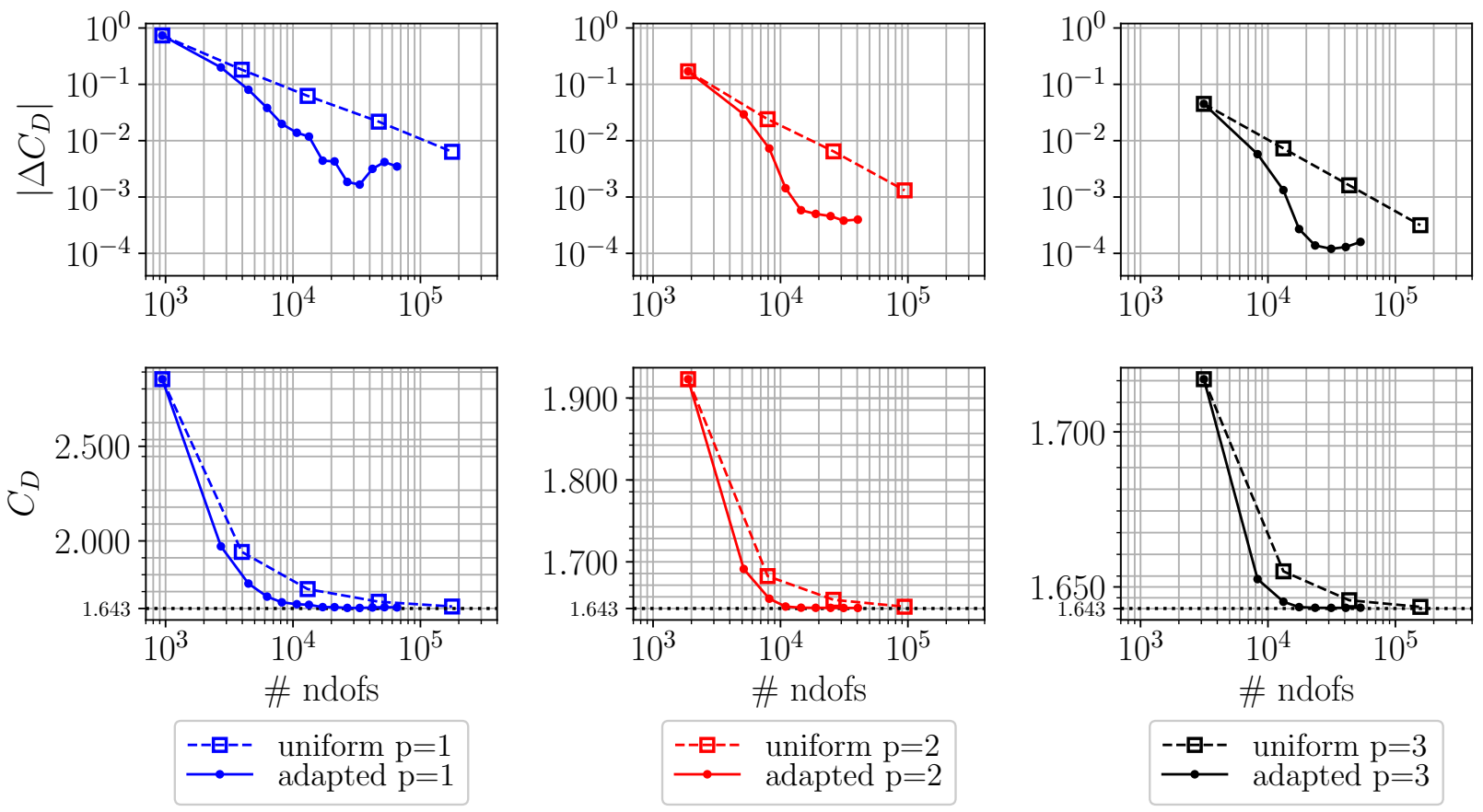

Fig. 3 Steady square cylinder flow at $R e=40$. Comparison between the convergence history of the drag coefficient for $h$-adapted meshes and uniformly refined meshes in $p=1, p=2, p=3 .\left|C_{D}-C_{D_{\text {ref }}}\right|$ vs. the number of degrees of freedom (top) and $C_{D}$ vs. the number of degrees of freedom (bottom)

seven, four, and three adaptation steps are sufficient for $p=1, p=2$ and $p=3 h$-adapted simulations to reach a level of error on the drag coefficient lower than that corresponding to the most uniformly refined meshes.

We observe that for all three polynomial degrees, after reaching a minimum error level (around $10^{-3}$ for $p=1$, lower than $10^{-3}$ for $p=2$ and around $10^{-4}$ for $p=3$ ), the value of the error stabilizes. This behaviour can be explained by the fact that in this adaptation process the minimum size of the element is fixed, and this minimum size drives the error on characteristic quantities. Moreover, the singular nature of the studied configuration, the reference quantity still presents some uncertainties.

In figure 4 the quality of the results for $p=1$ is assessed by comparing streamwise momentum profiles in the wake corresponding to the initial mesh, the reference mesh and different steps of the adaptation. The profiles are computed at the center line behind the body, and at three streamwise locations behind the square cylinder, $x / L=1.5,2.5,4.5$. The improvement of the flow field description on the adapted meshes with respect to the initial one, achieved in all three adaptations performed with $p=1, p=2$ and $p=3$, is here highlighted. The simulation results obtained on the initial meshes show strong discrepancies with respect to the reference solution, while an excellent agreement is recovered thanks to the $h$-adaptation process. Tables 2 presents the drag coefficient error for $h$-adapted and uniformly refined meshes with an equivalent number of DoFs. The error values for $2 \cdot 10^{4} \mathrm{DoFs}$, in the uniformly refined cases, are deduced via interpolation from the available data. For the $h$-adapted case, which does not show a linear behaviour of the error, the values with the adaptive steps with the closest number of freedom to $2 \cdot 10^{4}$ are retained. The adaptive algorithm, for the three polynomial degrees considered, allows for the adaptations to reach an accuracy level approximately ten times higher than the respective uniformly refined simulations, with an equivalent number of DoFs.

\begin{tabular}{l|c|c|c}
\hline & $p=1$ & $p=2$ & $p=3$ \\
\hline uniformly refined & $4.39 \cdot 10^{-2}$ & $8.66 \cdot 10^{-3}$ & $4.31 \cdot 10^{-3}$ \\
h-adapted & $4.29 \cdot 10^{-3}$ & $4.58 \cdot 10^{-4}$ & $1.38 \cdot 10^{-4}$
\end{tabular}

Table 2 Steady square cylinder flow at $R e=40$. Errors on $C_{D}$ for approximately $2 \cdot 10^{4}$ degrees of freedom for $h$-adapted and uniformly refined meshes in $p=1, p=2, p=3$. 

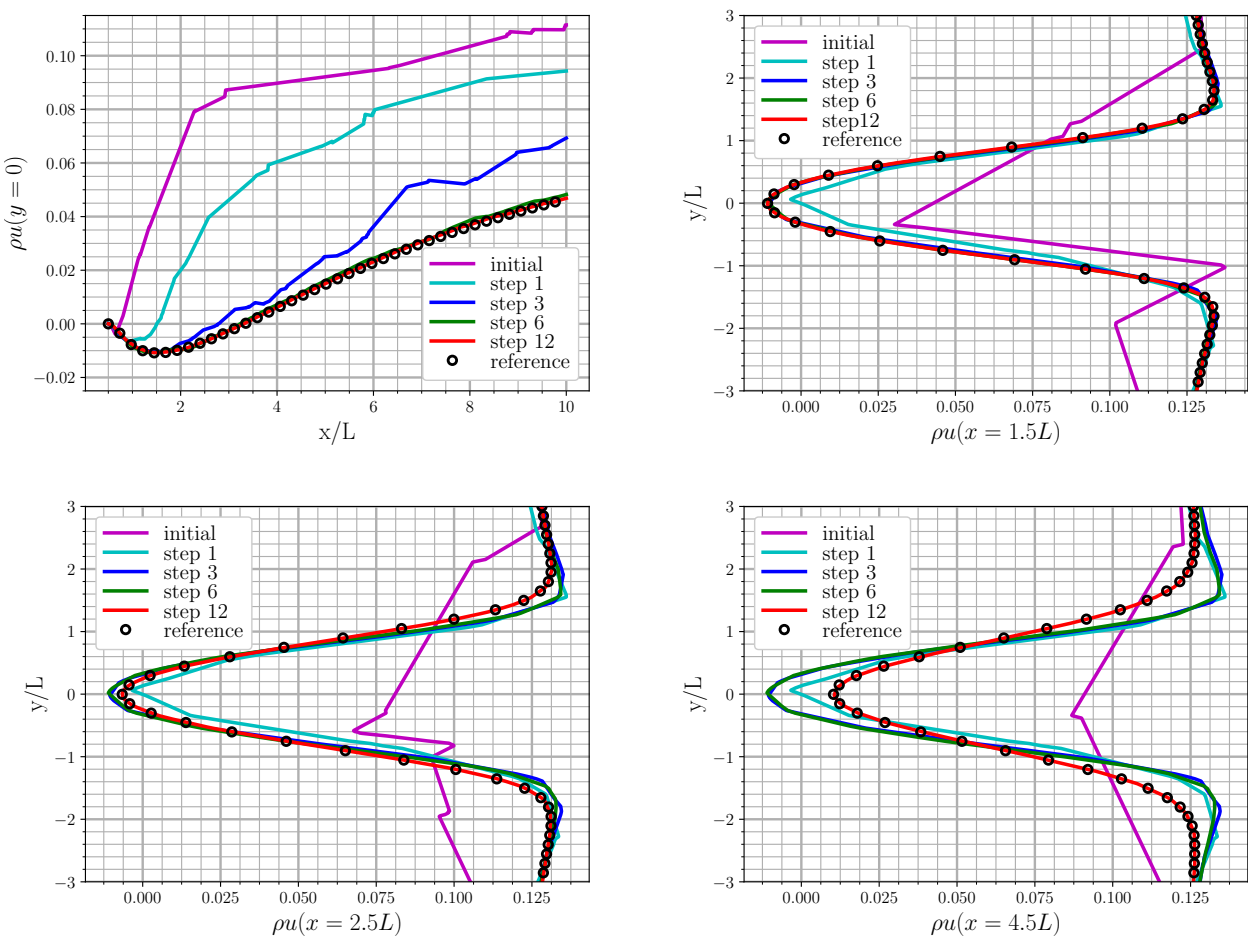

Fig. 4 Steady square cylinder at $R e=40$. Profiles of the $\mathbf{x}$-momentum on the center line $y=0$ behind the cylinder (top left), and at locations $x=1.5 \mathrm{~L}$ (top right), $x=2.5 \mathrm{~L}$ (bottom left), $x=4.5 \mathrm{~L}$ (bottom right). Comparisons between the initial mesh, the adapted meshes and the reference mesh for $p=1$.

In figure 5 the influence of the minimal size parameter $h_{\min }$ on the behavior of the adaptation process is studied, as it can be expected to be important for test cases showing strong geometrical singularities. Here we show the variation of the drag coefficient $C_{D}$ (right) and its decomposition in pressure $C_{\text {D pres }}$ (left) and friction $C_{D f \text { ric }}$ (middle) contribution. We notice that the prescription of a high $h_{\min }$ causes an overestimation of the friction component of the drag and an underestimation of the pressure component. In contrast, as can be seen from the figure 5, the minimum size seems to have a negligible influence on $C_{D}$. This behaviour comes from the cancellation of the errors due to the combination of the pressure and friction coefficients.

As an example, if we consider a value of $h_{\text {min }}=10^{-2}$ the pressure and friction drag coefficient present an error of the order of $10^{-2}$ with respect to the reference quantities. The combination of the two errors leads to an error on the total drag coefficient of the order of $10^{-4}$, similar to the error on the $C_{D}$ obtained with a simulation with $h_{\text {min }}=10^{-4}$. This misleading behaviour the total $C_{D}$ explains the necessity to analyze also the pressure and friction contributions to the drag.

The final simulations showed in figure 3 are performed with a $h_{\min }=10^{4} \mathrm{~L}$ which proved not to be affecting the convergence of the adaptation procedure. For values below a certain threshold of $h_{m i n}$, decreasing this parameter does not have relevant influence anymore on the adaptation process.

\begin{tabular}{l|l|l|l|l|l|l}
\hline$h_{\min }$ & $1 \cdot 10^{-2} L$ & $5 \cdot 10^{-3} L$ & $1 \cdot 10^{-3} L$ & $3 \cdot 10^{-4} L$ & $1 \cdot 10^{-4} L$ & uniform $5 \cdot 10^{-3} L$ \\
\hline$C_{\text {D pres }}$ & 1.4027 & 1.4074 & 1.4137 & 1.4159 & 1.4172 & 1.4088 \\
$C_{D \text { fric }}$ & 0.2399 & 0.2359 & 0.2297 & 0.2271 & 0.2257 & 0.2365 \\
$C_{D}$ & 1.6425 & 1.6433 & 1.6434 & 1.6430 & 1.6428 & 1.6452
\end{tabular}

Table 3 Steady square cylinder flow at $R e=40$. Values of $C_{D \text { pres }}, C_{D \text { f ric }}$ and $C_{D}$ for approximately $10^{5}$ degrees of freedom for $h$-adapted and uniformly refined meshes for different values of $h_{\min }$ for $p=2$ simulations. 

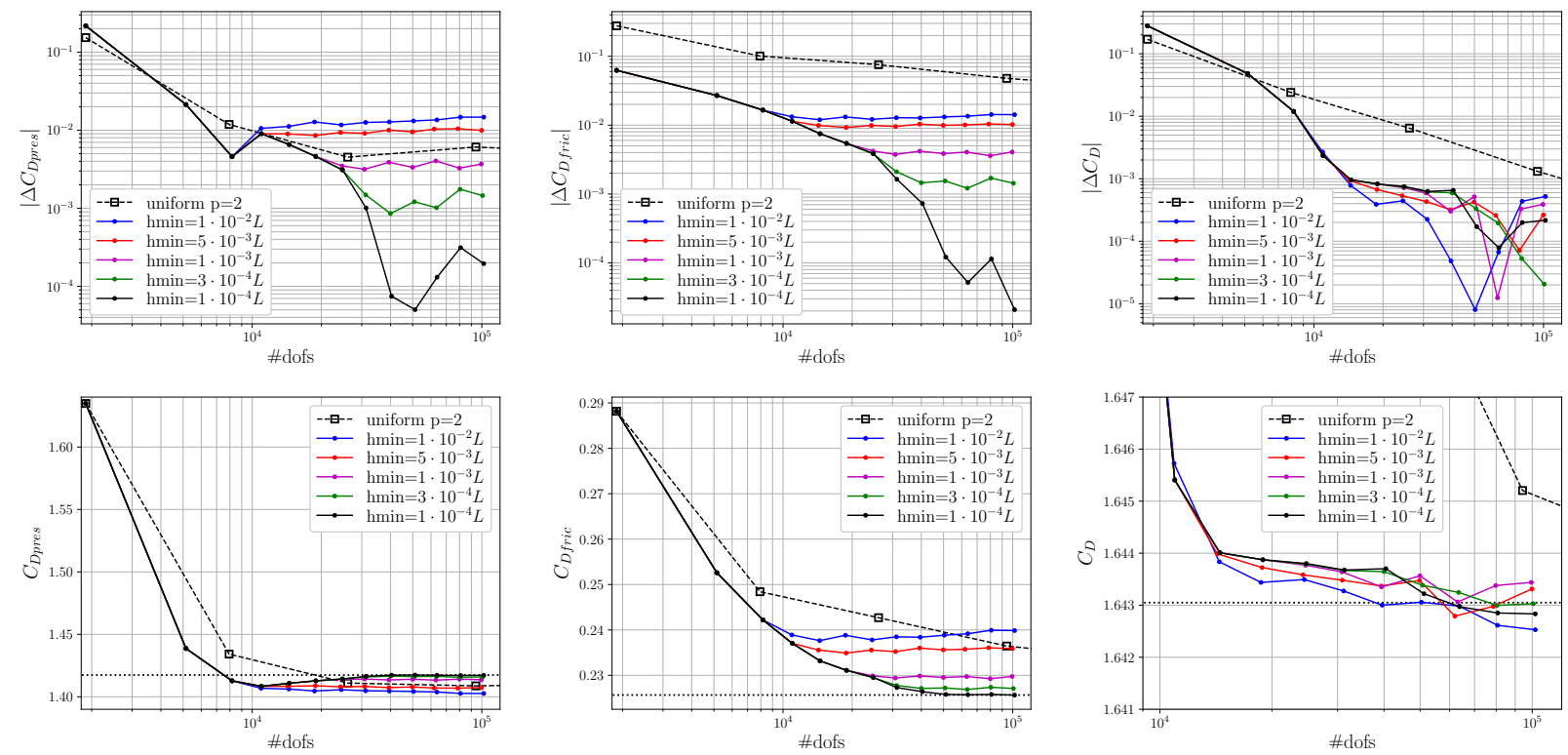

Fig. 5 Steady square cylinder flow at $R e=40 . C_{D \text { pres }}, C_{D f \text { ric }}, C_{D}$ vs number of DOFs for different values of $h_{\text {min }}$. Errors on the top and values on the bottom.

\section{Laminar steady flow past a NACA0012 airfoil at Reynolds $=5000$, Mach $=0.5$ and $\alpha=1^{\circ}$}

The $h$-adaptive algorithm is assessed in the present section for a flow representative of aerodynamic applications. We consider the steady, subsonic and viscous flow around the NACA0012 airfoil which is a well-known test case used in CFD for validation purposes (turbulence models, shape optimization, numerical schemes), and among others for steady mesh adaptation [65-68].

The configuration studied here has a free-stream Mach number of $M_{\infty}=0.5$, angle of attack $\alpha=1^{\circ}$ and Reynolds number of $R e=5000$. The main feature of this flow is the thin, laminar boundary layer developing over the airfoil.

Reference results are obtained from a $p=3$ discretization highly refined around the body, with a 443273 elements mesh obtained via uniform refinement (roughly $4.5 \times 10^{6} \mathrm{DoFs}$ ). Those results compare favorably with other results present in the literature, as seen in Table 4

\begin{tabular}{l|l|l|l|l}
\hline & \multicolumn{1}{|c|}{$C_{D}$} & \multicolumn{1}{|c|}{$C_{\text {Df ric }}$} & \multicolumn{1}{|c}{$C_{\text {Dpres }}$} & \multicolumn{1}{c}{$C_{L}$} \\
\hline Swanson et al. (NASA report) [69] & 0.055980 & 0.0327577 & 0.023222 & 0.0184635 \\
Yano and Darmofal [67] & 0.0553167 & - & - & 0.018274 \\
Balan et Al [68] & 0.0553168 & - & - & - \\
\hline current & 0.0556622 & 0.0328013 & 0.0228610 & 0.0199830 \\
\hline
\end{tabular}

Table 4 Steady NACA0012 flow at $R e=5000$. Integral flow quantities in the literature and for the present reference.

The mesh shown in the left in figure 6 with 789 triangular elements is used as the initial mesh, for the computations with the three polynomial degrees considered. It is a C-type mesh, centered at the airfoil mid chord with a radius of 80 chords and 100 chords in the rear region. In order to assess the effect of the polynomial degree on the error convergence rate, the adaptations are performed on different spatial discretizations $(p=1,2,3)$.

Figure 6 shows that the adaptation yields a concentration of the refinement in the boundary layer region around the airfoil, the stagnation region near the leading edge and the wake in the region downstream of the airfoil.

Figures 7 and 8 show comparisons respectively of the errors on the drag coefficient and on the lift coefficient for uniformly refined and $h$-adapted meshes. In early refinement steps we notice a higher refinement than the prescribed one: in fact the equation 28, based on the hypothesis of isotropy of the mesh can yield an excess of elements compared 

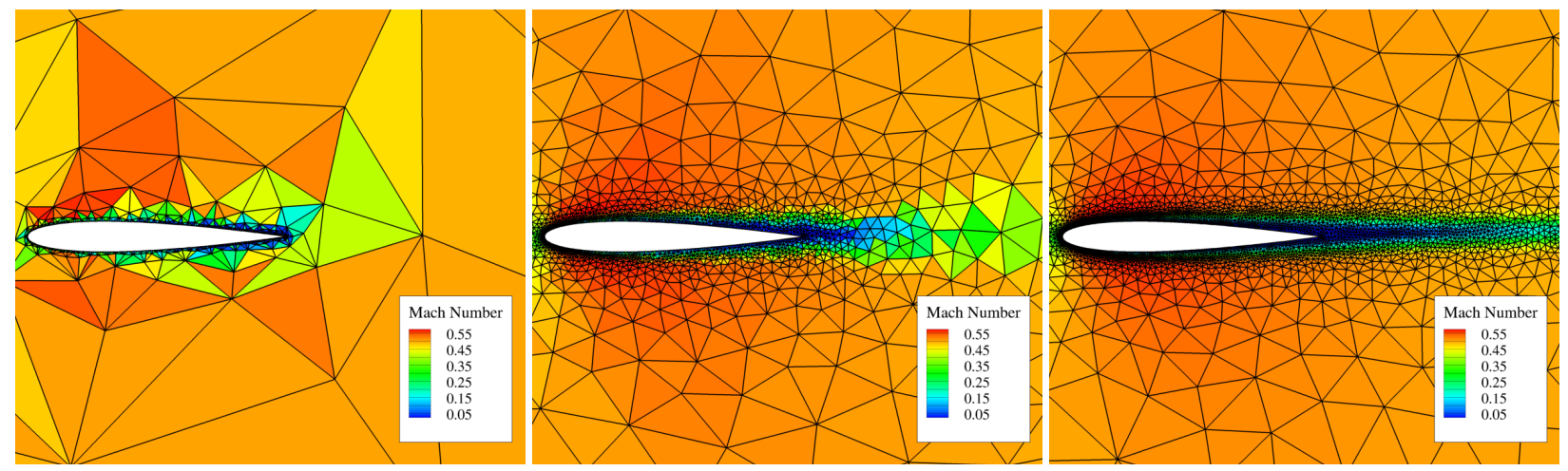

Fig. 6 Steady NACA0012 flow at $R e=5000$. Zoom of the Mach number iso-contours of the cell-averaged solution on the initial mesh ( 789 elements), on the $3^{\text {rd }}$ adapted mesh (3750 elements) and on the $6^{\text {th }}$ adapted mesh (8750 elements), for the $p=2$ simulations.

to the number requested when the mesh is very coarse and distorted. After a few steps the right behaviour is recovered, with a smaller increment of number of elements from one adaptation to the next.

Adapted simulations reach the same level of error compared to the corresponding most uniformly refined mesh with about the tenth of number of DoFs, for both $\left|\Delta C_{D}\right|$ and $\left|\Delta C_{L}\right|$.
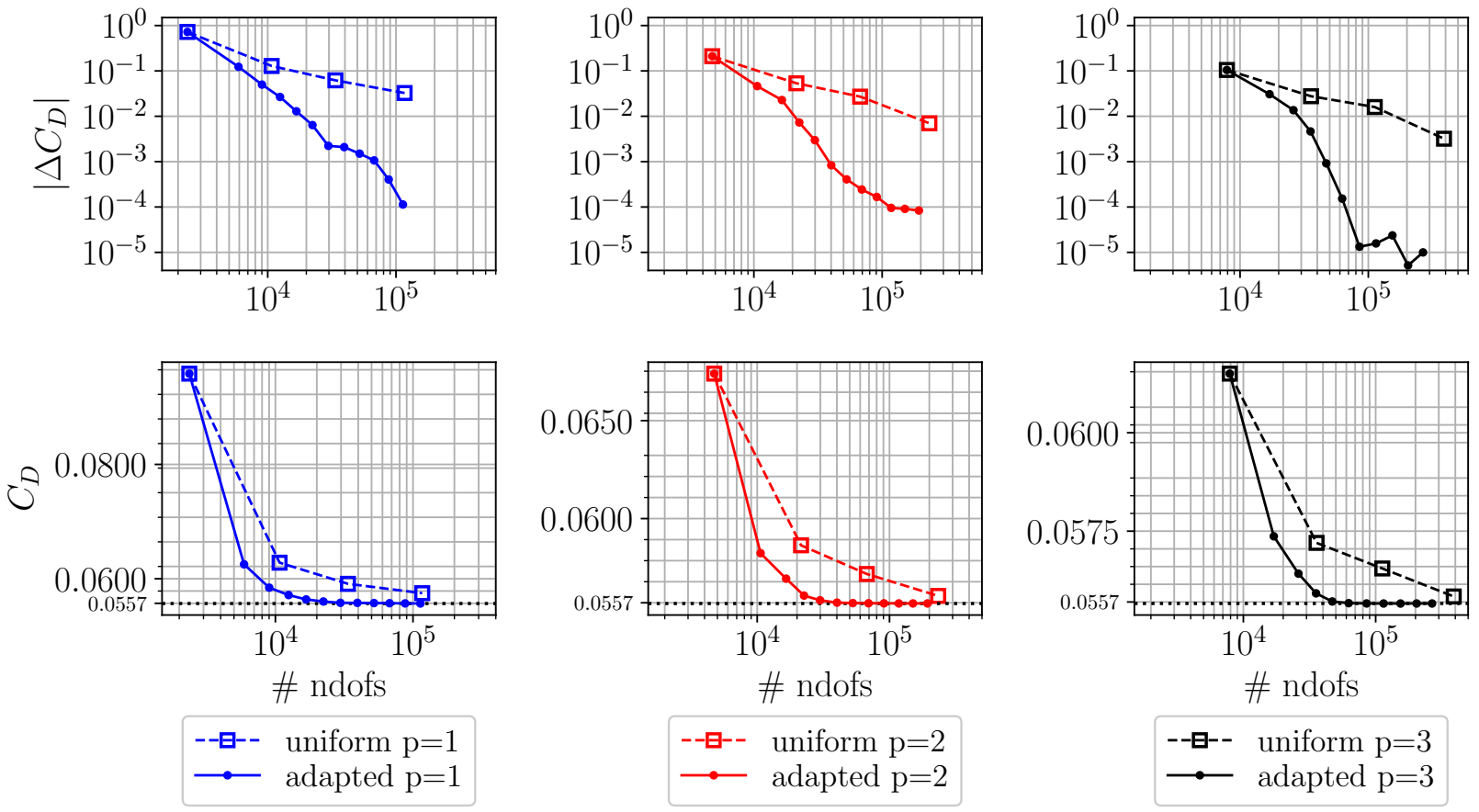

Fig. 7 Steady NACA0012 flow at $R e=5000$. Comparisons between the convergence history of the drag coefficient for $h$-adapted meshes and uniformly refined meshes in $p=1, p=2, p=3 .\left|C_{D}-C_{D_{\text {ref }}}\right|$ vs. the number of degrees of freedom (top) and $C_{D}$ vs. the number of degrees of freedom (bottom)

A comparison of error levels between adapted and uniform simulations featuring the same number of DoFs $4 \cdot 10^{4}$ is provided in Tables 5 and 6 For higher order adapted simulations, $p=2$ and $p=3$ we observe that the error in the drag and lift coefficients is reduced by almost two orders of magnitude with respect to the uniformly refined simulations. The decrease for second order $p=1$ simulations is lower: that might be due to the fact that we are farther from the converged solution, and to the lower performances of the error estimator for $p=1$.

The influence of the $f_{\mathcal{C}}$, which controls the complexity of the refinement is assessed in figure 9 . The algorithm 

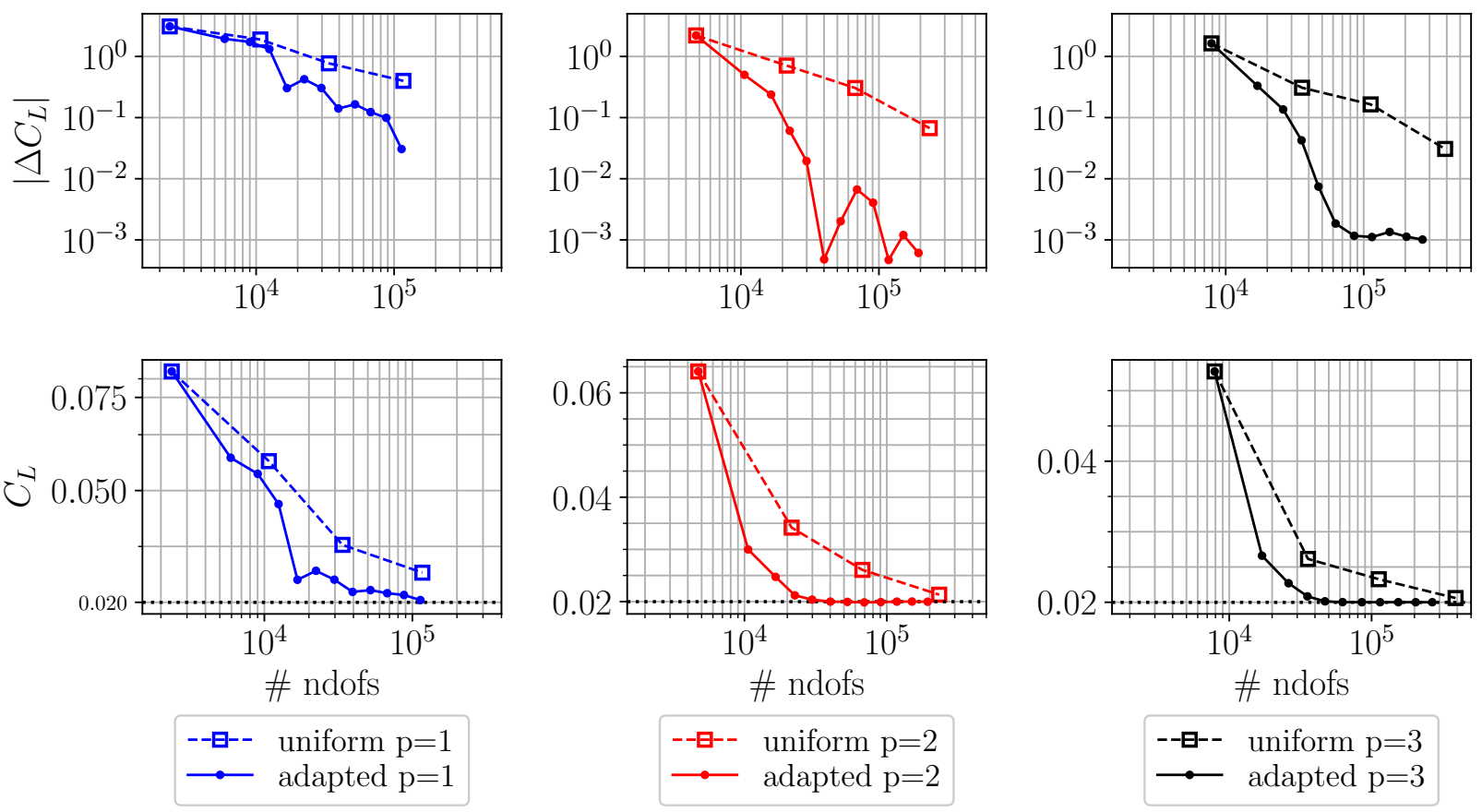

Fig. 8 Steady NACA0012 flow at $R e=5000$. Comparisons between the convergence history of the lift coefficient for $h$-adapted meshes and uniformly refined meshes in $p=1, p=2, p=3$. $\left|C_{L}-C_{L_{\text {ref }}}\right|$ vs. the number of degrees of freedom (top) and $C_{L}$ vs. the number of degrees of freedom (bottom)

\begin{tabular}{l|c|c|c}
\hline & $p=1$ & $p=2$ & $p=3$ \\
\hline uniformly refined & $5.67 \cdot 10^{-2}$ & $3.69 \cdot 10^{-2}$ & $2.62 \cdot 10^{-2}$ \\
h-adapted & $3.14 \cdot 10^{-3}$ & $9.56 \cdot 10^{-4}$ & $8.52 \cdot 10^{-4}$
\end{tabular}

Table 5 Steady NACA0012 flow at $R e=5000$. Errors on $C_{D}$ for approximately $4 \cdot 10^{4}$ degrees of freedom for $h$-adapted and uniformly refined meshes in $p=1, p=2, p=3$.

\begin{tabular}{l|c|c|c}
\hline & $p=1$ & $p=2$ & $p=3$ \\
\hline uniformly refined & $7.05 \cdot 10^{-1}$ & $4.48 \cdot 10^{-1}$ & $2.90 \cdot 10^{-1}$ \\
h-adapted & $3.84 \cdot 10^{-1}$ & $5.29 \cdot 10^{-3}$ & $6.44 \cdot 10^{-3}$
\end{tabular}

Table 6 Steady NACA0012 flow at $R e=5000$. Errors on $C_{L}$ for approximately $4 \cdot 10^{4}$ degrees of freedom for $h$-adapted and uniformly refined meshes in $p=1, p=2, p=3$.

is tested with four different values of the refinement factor $f_{C}$ from 1.1 to 2 , and the impact of this parameter on the convergence history of the $C_{L}$ and $C_{D}$ values is assessed. All choices lead the integral quantities to convergence towards the same values, showing a low sensitivity to this parameter. The parameter, however, affects the rate of convergence in terms of number of degrees of freedom. In particular, a higher value of the refinement factor $f_{C}$ leads to a lower number of elements being selected for refinement at each step of the adaptive algorithm, therefore increasing the number of refinement steps in order to achieve the same resolution. At the same time, despite the fact that a very small $f_{C}$ makes the algorithm converge to the same value with much less degrees of freedom, it needs more adaptation steps to achieve convergence. This process can be time consuming. In fact each simulation is initialized from the solution obtained at the previous refinement level interpolated to the new mesh, but the residuals of the Navier-Stokes equations need to decrease at every step to a suitable low residual threshold to converge to a steady state solution. The choice of the increment in terms of number of nodes (and elements) from one step to another can thus be seen as a trade-off between 
a low number of adaptation steps and a low computational time. For this reason, in the present work we use the value $f_{C}=1.3$, which proved to be an efficient choice to ensure a good convergence and a low number of iterations.
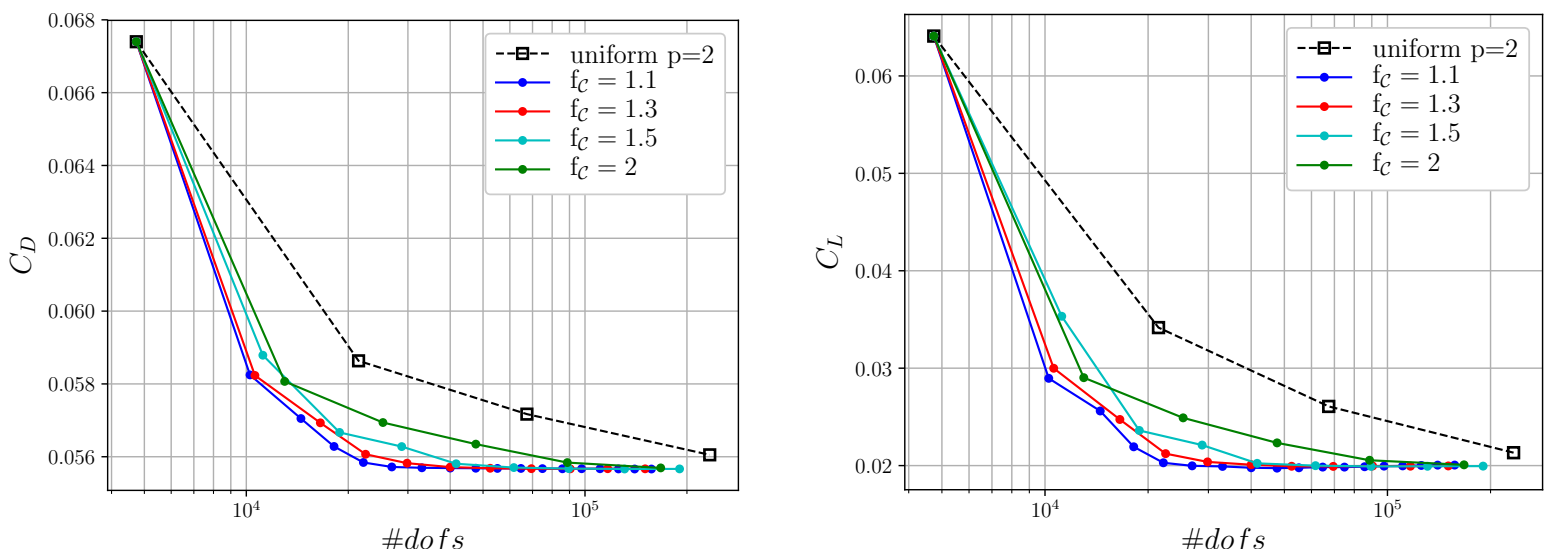

Fig. 9 NACA0012 flow at $R e=5000$. Sensitivity of $C_{D}$ and $C_{L}$ mesh convergence for different values of $f_{C}$.

\section{B. Unsteady laminar flow past a circular cylinder at Reynolds $=100$}

The h-adaptive algorithm is validated on the laminar unsteady flow past a circular cylinder at Reynolds $R e=100$ based on the cylinder diameter $D$ and $M a c h=0.1$, already been studied for adaptive algorithms [59, 70]. The main feature of this flow consists in the vortices created behind the cylinder, which detach periodically from either side of the body forming a Von Kármán vortex street.

The parameters considered for the adaptation simulations featuring the polynomial degrees $p=1, p=2$ and $p=3$ are the same used for steady adaptations in the previous subsection. Exceptions are the higher $h_{\text {min }}=10^{-2} D$ for all unsteady simulations, and a high value of the refinement factor $f_{C}=3$ used for $p=1$.

Initial meshes are created with MMG2D by providing a metric and forcing the location of the mesh nodes on the wall. Unlike the steady cases where a trivial constant metric field was used, we chose here to set gradual sizes varying with the radius from the body to the far field. In fact a bad resolution of the initial mesh behind the body prevents the flow around the cylinder from shedding due to high dissipation. The cylinder would require then many adaptive (and uniform) steps to grant a shedding configuration.

The initial mesh of 522 elements is shown in figure 10 at the left and the reference results used in this study have been performed on a $p=2$ mesh with 340161 elements ( 2 millions of degrees of freedom). The values obtained are compared with those present in the literature in table 7 .

The time integration is performed by using a classical explicit fourth-order Runge-Kutta scheme with a constant time step. For each simulation the time step is chosen as the highest time step granting stability. The used time steps varies from $\Delta t=10^{-2}$ of the coarsest $p=1$ simulation to $\Delta t=10^{-4}$ of the most refined $p=3$.

The statistics on the error estimator are gathered after the periodic state is reached, over one shedding period, and sampled with a constant sampling time step of $\Delta t=10^{-2}$. The periodic state is considered to be reached when the variation in the Strouhal number, the root mean square of the lift and the average drag coefficient measured between two shedding cycles are respectively $\Delta C D_{\text {avg }}<10^{-5}, \Delta C L_{r m s} 10^{-5}$, Strouhal $<10^{-6}$.

In figure 11 we show the convergence history of the root mean square of the lift coefficient and the average of the drag coefficient for h-adapted and uniformly refined simulations. The similar convergence behaviour of $p=2$ and $p=3$ is due to the fact that linear elements are used to discretize the surface, leading to a loss of the benefits of high-order accuracy for flows configurations where the geometry is fundamental.

The reason why higher refinement factor is needed for $p=1$ to achieve faster convergence than uniform meshes is to address to geometrical reasons: the initial mesh, despite being of better quality than the initial meshes exploited for steady cases, turned out being too coarse on the surface to well capture the geometry. The error estimator does not activate uniformly over the surface of the body, and the coarse linear geometry behind the cylinder, where the error estimator is lower, is not capable of representing the right curved geometry. 


\begin{tabular}{l|l|l|l|l}
\hline & $C_{\text {Davg }}$ & $C_{\text {Drms }}$ & $C_{\text {Lrms }}$ & Strouhal \\
\hline Baranyi et al. [71] & & 0.0064 & 0.228 & \\
\hline Naddei et al. [59] & 1.326 & - & 0.2266 & 0.1638 \\
\hline Norberg (exp) [72] & - & - & 0.227 & 0.164 \\
\hline current & 1.3292 & 0.00635 & 0.22731 & 0.16408 \\
\hline
\end{tabular}

Table 7 Unsteady circular cylinder flow at $R e=100$. Integral flow quantities in the literature and for the present reference.
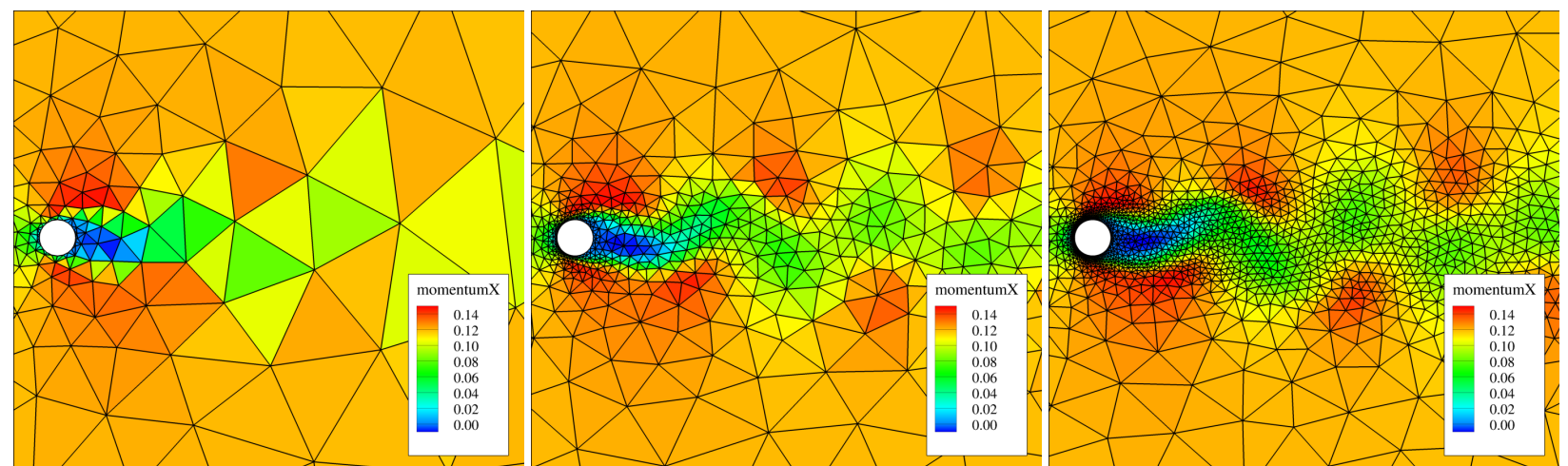

Fig. 10 Unsteady circular cylinder flow at $R e=100$. Zoom of the $x$-momentum iso-contours of the cell-averaged solution on the initial mesh (522 elements), on the $3^{\text {rd }}$ adapted mesh (1746 elements) and on the $8^{\text {th }}$ adapted mesh (6453 elements), for the $p=3$ simulations.

Despite this, we show how the algorithm is capable of reaching the right values of $C_{\text {Davg }}$ and $C_{L r m s}$ with less degrees of freedom than the uniform meshes.

A zoom of adapted meshes is shown in figure 10 after 3 adaptive steps (middle, 1746 elements) and after 8 adaptations (left, 6453 elements) for a $p=3$ discretization. The contour of the momentum in the longitudinal direction is represented at different instants of the shedding period. Adapted meshes well capture the flow around the body and the wake which develops behind the cylinder.

Finally we assess the robustness of the statistics gathering method of the error estimator. We perform in figure 12 a comparison between the $\mathcal{L}^{1}$ norm, which is the average of the error estimator in each element for one shedding period (which is the method used in the results presented previously), and the $\mathcal{L}^{\infty}$ norm, corresponding to the maximum value of the error estimator in the period. The behaviour in early stages of the adaptive process of the quantities of interest $C_{\text {Drms }}$ and $C_{\text {Lrms }}$ show a slight improvement using the $\mathcal{L}^{1}$ norm, while the overall convergence is very similar for the two types of statistics.

\section{Conclusions}

In the present paper, a $h$-adaptation strategy suitable to discontinuous Galerkin methods on unstructured meshes has been proposed for solving fluid flow problems. A novel error estimator based on both the measure of the energy contained in the highest order polynomial modes and the jumps at the element interfaces has been assessed in the framework of static isotropic metric-based $h$-adaptation. $h$-adapted DG computations of two laminar steady flows, past a square cylinder and a NACA0012 airfoil, and the laminar unsteady flow past a circular cylinder, have been performed with polynomial degrees $p=1, p=2$ and $p=3$. The overall adaptation strategy is found to capture accurately the zones in which the solution needs higher refinement and zones already well refined which allow for coarsening. This yields a significant reduction in terms of number of degrees of freedom to reach a given error threshold, compared to the non-adapted simulations. Future work will address the topic of $h p$-adaptation as well as the application of the methodology to LES configurations with the development of ad-hoc error indicators. 

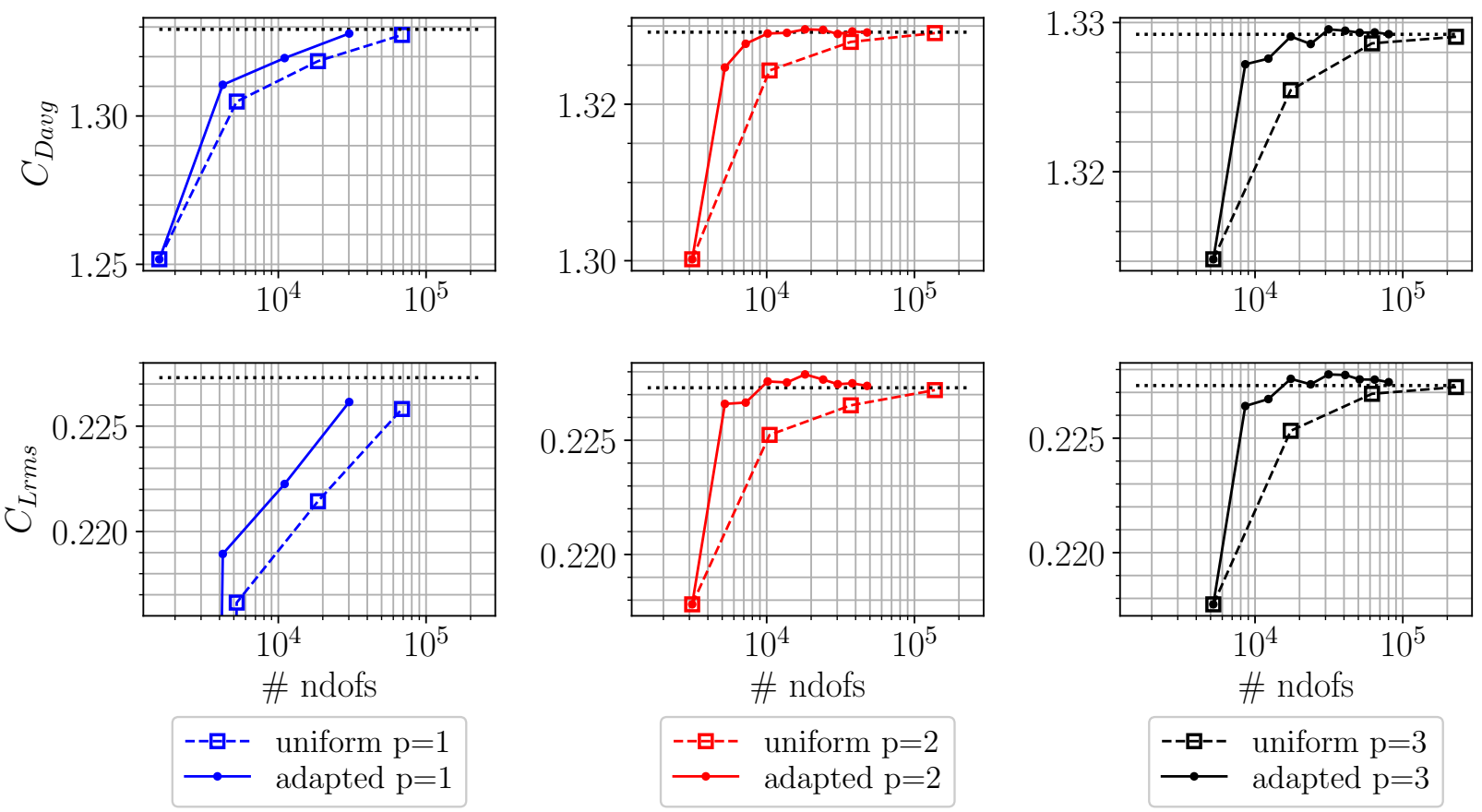

Fig. 11 Unsteady circular cylinder flow at $R e=100$. Comparisons between the convergence history of the average drag coefficient and the $r m s$ of the lift coefficient for $\boldsymbol{h}$-adapted meshes and uniformly refined meshes in $p=1, p=2, p=3$. $C_{D a v g}$ vs. the number of degrees of freedom (top) and $C_{L r m s}$ vs. the number of degrees of freedom (bottom)
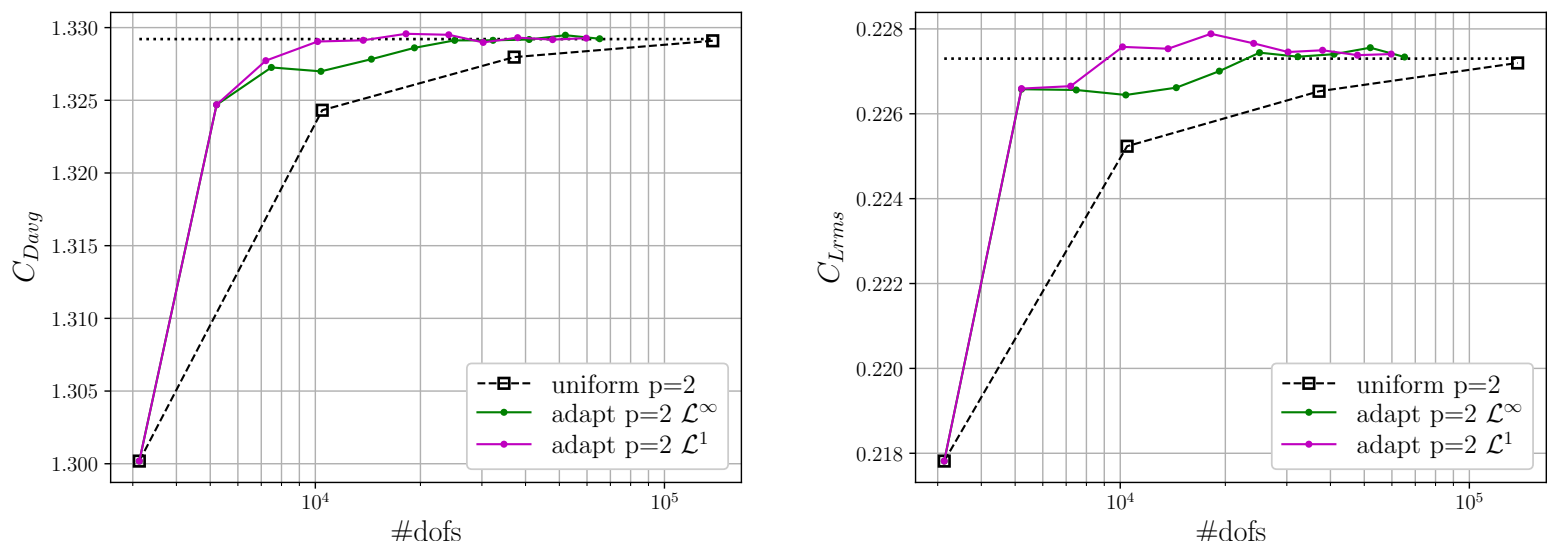

Fig. 12 Unsteady circular cylinder flow at $R e=100$. Comparison between unsteady h-adaptive simulation using the $\mathcal{L}_{1}$ and the $\mathcal{L}_{\infty}$ norms of the error estimator over one period.

\section{Acknowledgments}

The authors would like to thank Dr Fabio Naddei from ONERA for fruitful discussions, as well as Dr Tobias Leicht from DLR for the development of the high-order projection functionalities in CODA and Dr Algiane Froehly from INRIA for her help regarding MMG features. This work was performed using HPC resources from GENCI-CINES (Grant 2020-A0082A11470) and internal ONERA HPC resources. 


\section{References}

[1] Karniadakis, G., and Sherwin, S., Spectral/hp element methods for computational fluid dynamics, Oxford University Press, 2013.

[2] Dolejší, V., and Feistauer, M., "Discontinuous galerkin method," Analysis and Applications to Compressible Flow. Springer Series in Computational Mathematics, Vol. 48, 2015.

[3] Reed, W. H., and Hill, T., "Triangular mesh methods for the neutron transport equation," Tech. rep., Los Alamos Scientific Lab., N. Mex.(USA), 1973.

[4] Cockburn, B., Discontinuous Galerkin methods for convection-dominated problems, Springer, 1999.

[5] Mavriplis, C., "A posteriori error estimators for adaptive spectral element techniques," Proceedings of the Eighth GAMMConference on Numerical Methods in Fluid Mechanics, Springer, 1990, pp. 333-342.

[6] Persson, P.-O., and Peraire, J., "Sub-cell shock capturing for discontinuous Galerkin methods," 44th AIAA Aerospace Sciences Meeting and Exhibit, 2006, p. 112.

[7] Gassner, G., Altmann, C., Hindenlang, F., Staudenmeier, M., and Munz, C., "Explicit Discontinuous Galerkin Schemes with Adaptation in Space and Time," 36th CFD/ADIGMA course on hp-adaptive and hp-multigrid methods, VKI LS, 2009.

[8] Remacle, J.-F., Flaherty, J. E., and Shephard, M. S., "An adaptive discontinuous Galerkin technique with an orthogonal basis applied to compressible flow problems," SIAM review, Vol. 45, No. 1, 2003, pp. 53-72.

[9] Dolejší, V., "hp-DGFEM for nonlinear convection-diffusion problems," Mathematics and Computers in Simulation, Vol. 87, 2013, pp. 87-118.

[10] Leicht, T., and Hartmann, R., "Error estimation and hp-adaptive mesh refinement for discontinuous Galerkin methods," Adaptive high-order methods in computational fluid dynamics, World Scientific, 2011, pp. 67-94.

[11] Wang, L., and Mavriplis, D. J., "Adjoint-based h-p adaptive discontinuous Galerkin methods for the 2D compressible Euler equations," Journal of Computational Physics, Vol. 228, No. 20, 2009, pp. 7643-7661.

[12] Kuru, G., de la Llave Plata, M., Couaillier, V., Abgrall, R., and Coquel, F., "An adaptive variational multiscale discontinuous Galerkin method for large eddy simulation," 54th AIAA Aerospace Sciences Meeting, 2016, p. 0584.

[13] Baker, T. J., "Mesh adaptation strategies for problems in fluid dynamics," Finite Elements in Analysis and Design, Vol. 25, No. 3-4, 1997, pp. 243-273.

[14] Remacle, J.-F., Li, X., Chevaugeon, N., and Shephard, M. S., "Transient Mesh Adaptation Using Conforming and Non Conforming Mesh Modifications.” IMR, 2002, pp. 261-272.

[15] Ceze, M., and Fidkowski, K., “Anisotropic hp-Adaptation Framework for Functional Prediction,” AIAA Journal, 2012. https://doi.org/10.2514/1.J051845

[16] Leicht, T., and Hartmann, R., "Error estimation and anisotropic mesh refinement for 3d laminar aerodynamic flow simulations," Journal of Computational Physics, Vol. 229, No. 19, 2010, pp. 7344-7360.

[17] Wackers, J., Deng, G., Guilmineau, E., Leroyer, A., Queutey, P., and Visonneau, M., "Combined refinement criteria for anisotropic grid refinement in free-surface flow simulation," Computers \& Fluids, Vol. 92, 2014, pp. $209-222$.

[18] Arnold, D. N., Mukherjee, A., and Pouly, L., "Locally adapted tetrahedral meshes using bisection," SIAM Journal on Scientific Computing, Vol. 22, No. 2, 2000, pp. 431-448.

[19] Rivara, M.-C., and Iribarren, G., "The 4-triangles longest-side partition of triangles and linear refinement algorithms," Mathematics of Computation, Vol. 65, No. 216, 1996, pp. 1485-1502.

[20] Zhang, L.-B., "A parallel algorithm for adaptive local refinement of tetrahedral meshes using bisection," Numer. Math.: Theory, Methods and Applications, Vol. 2, 2009, pp. 65-89.

[21] Loseille, A., and Alauzet, F., "Continuous mesh framework part I: well-posed continuous interpolation error," SIAM Journal on Numerical Analysis, Vol. 49, No. 1, 2011, pp. 38-60.

[22] Dapogny, C., Dobrzynski, C., and Frey, P., “Three-dimensional adaptive domain remeshing, implicit domain meshing, and applications to free and moving boundary problems," Journal of computational physics, Vol. 262, 2014, pp. 358-378. 
[23] Yano, M., Modisette, J., and Darmofal, D., "The importance of mesh adaptation for higher-order discretizations of aerodynamic flows," 20th AIAA Computational Fluid Dynamics Conference, 2011, p. 3852.

[24] Li, X., Shephard, M. S., and Beall, M. W., “3D anisotropic mesh adaptation by mesh modification,” Computer methods in applied mechanics and engineering, Vol. 194, No. 48-49, 2005, pp. 4915-4950.

[25] Daviller, G., Brebion, M., Xavier, P., Staffelbach, G., Müller, J.-D., and Poinsot, T., "A mesh adaptation strategy to predict pressure losses in les of swirled flows," Flow, Turbulence and Combustion, Vol. 99, No. 1, 2017, pp. 93-118.

[26] Park, M. A., “Adjoint-based, three-dimensional error prediction and grid adaptation,” AIAA journal, Vol. 42, No. 9, 2004 , pp. $1854-1862$.

[27] Fidkowski, K. J., and Darmofal, D. L., "Review of output-based error estimation and mesh adaptation in computational fluid dynamics," AIAA journal, Vol. 49, No. 4, 2011, pp. 673-694.

[28] Frey, P.-J., and Alauzet, F., "Anisotropic mesh adaptation for CFD computations," Computer methods in applied mechanics and engineering, Vol. 194, No. 48-49, 2005, pp. 5068-5082.

[29] Castro-Díaz, M., Hecht, F., Mohammadi, B., and Pironneau, O., "Anisotropic unstructured mesh adaption for flow simulations," International Journal for Numerical Methods in Fluids, Vol. 25, No. 4, 1997, pp. 475-491.

[30] Alauzet, F., and Loseille, A., "A decade of progress on anisotropic mesh adaptation for computational fluid dynamics," Computer-Aided Design, Vol. 72, 2016, pp. 13-39.

[31] Remacle, J.-F., Li, X., Shephard, M. S., and Flaherty, J. E., "Anisotropic adaptive simulation of transient flows using discontinuous Galerkin methods," International Journal for Numerical Methods in Engineering, Vol. 62, No. 7, 2005, pp. 899-923.

[32] Dolejší, V., "Anisotropic hp-adaptive method based on interpolation error estimates in the Lq-norm," Applied Numerical Mathematics, Vol. 82, 2014, pp. 80-114.

[33] Balan, A., Woopen, M., and May, G., "Adjoint-based hp-adaptivity on anisotropic meshes for high-order compressible flow simulations," Computers \& Fluids, Vol. 139, 2016, pp. 47-67.

[34] Rangarajan, A., May, G., and Dolejsi, V., “Adjoint-based anisotropic hp-adaptation for discontinuous Galerkin methods using a continuous mesh model," Journal of Computational Physics, Vol. 409, 2020, p. 109321.

[35] Coulaud, O., and Loseille, A., "Very high order anisotropic metric-based mesh adaptation in 3d," Procedia engineering, Vol. 163, 2016, pp. 353-365.

[36] Bernard, P.-E., Chevaugeon, N., Legat, V., Deleersnijder, E., and Remacle, J.-F., "High-order h-adaptive discontinuous Galerkin methods for ocean modelling," Ocean Dynamics, Vol. 57, No. 2, 2007, pp. 109-121.

[37] Hecht, F., "BAMG: bidimensional anisotropic mesh generator," User Guide. INRIA, Rocquencourt, Vol. 17, 1998.

[38] Loseille, A., and Lohner, R., "Anisotropic adaptive simulations in aerodynamics," 48th AIAA Aerospace Sciences Meeting Including the New Horizons Forum and Aerospace Exposition, 2010, p. 169.

[39] Michal, T., and Krakos, J., "Anisotropic mesh adaptation through edge primitive operations," 50th AIAA Aerospace Sciences Meeting including the New Horizons Forum and Aerospace Exposition, 2012, p. 159.

[40] Ibanez, D., and Shephard, M., "Mesh adaptation for moving objects on shared memory hardware," 25th International Meshing Roundtable, Sandia National Laboratories, 2016, pp. 1-5.

[41] Gorman, G. J., Rokos, G., Southern, J., and Kelly, P. H., “Thread-parallel anisotropic mesh adaptation,” New Challenges in Grid Generation and Adaptivity for Scientific Computing, Springer, 2015, pp. 113-137.

[42] Michal, T., Babcock, D., Kamenetskiy, D., Krakos, J., Mani, M., Glasby, R., Erwin, T., and Stefanski, D. L., "Comparison of fixed and adaptive unstructured grid results for drag prediction workshop 6," Journal of Aircraft, Vol. 55, No. 4, 2018 , pp. $1420-1432$.

[43] Alauzet, F., and Loseille, A., "Metrix User Guide. Error Estimates and Mesh Control for Anisotropic Mesh Adaptation,” 2009.

[44] Dobrzynski, C., and Frey, P., “Anisotropic Delaunay mesh adaptation for unsteady simulations," Proceedings of the 17th international Meshing Roundtable, Springer, 2008, pp. 177-194. 
[45] Benard, P., Balarac, G., Moureau, V., Dobrzynski, C., Lartigue, G., and d'Angelo, Y., "Mesh adaptation for large-eddy simulations in complex geometries," International journal for numerical methods in fluids, Vol. 81, No. 12, 2016, pp. 719-740.

[46] Bourasseau, S., "Contribution to a mesh refinement method based on the adjoint vector for the computation of aerodynamic outputs (Theses)," Université Nice Sophia Antipolis, 2015.

[47] Sadaka, G., Rakotondrandisa, A., Tournier, P.-H., Luddens, F., Lothodé, C., and Danaila, I., "Parallel finite-element codes for the simulation of two-dimensional and three-dimensional solid-liquid phase-change systems with natural convection," 2020.

[48] Pope, S. B., “Turbulent flows,”, 2001.

[49] Leicht, T., Jägersküpper, J., Vollmer, D., Schwöppe, A., Hartmann, R., Fiedler, J., and Schlauch, T., "DLR-Project Digital-X-Next Generation CFD Solver'Flucs'," CEAS Aeronautical Journal, 2016.

[50] Butcher, J. C., "Coefficients for the study of Runge-Kutta integration processes," Journal of the Australian Mathematical Society, Vol. 3, No. 2, 1963, p. 185-201. https://doi.org/10.1017/S1446788700027932

[51] Bassi, F., Botti, L., Colombo, A., Di Pietro, D. A., and Tesini, P., "On the flexibility of agglomeration based physical space discontinuous Galerkin discretizations," Journal of Computational Physics, Vol. 231, No. 1, 2012, pp. 45-65.

[52] Rusanov, V., "Calculation of interaction of non-steady schock waves with obstacles," J. Comp. Math. Phys, Vol. 1, 1961, pp. 267-279.

[53] Roe, P. L., “Approximate Riemann solvers, parameter vectors, and difference schemes," Journal of computational physics, Vol. 43, No. 2, 1981, pp. 357-372.

[54] Harten, A., and Hyman, J. M., "Self adjusting grid methods for one-dimensional hyperbolic conservation laws," Journal of computational Physics, Vol. 50, No. 2, 1983, pp. 235-269.

[55] Bassi, F., and Rebay, S., "A high-order accurate discontinuous finite element method for the numerical solution of the compressible Navier-Stokes equations," Journal of computational physics, Vol. 131, No. 2, 1997, pp. 267-279.

[56] Bassi, F., Rebay, S., Mariotti, G., Pedinotti, S., and Savini, M., "A high-order accurate discontinuous finite element method for inviscid and viscous turbomachinery flows," Proceedings of the 2nd European Conference on Turbomachinery Fluid Dynamics and Thermodynamics, Technologisch Instituut, Antwerpen, Belgium, 1997, pp. 99-109.

[57] Bassi, F., Crivellini, A., Rebay, S., and Savini, M., "Discontinuous Galerkin solution of the Reynolds-averaged Navier-Stokes and k- $\omega$ turbulence model equations," Computers \& Fluids, Vol. 34, No. 4-5, 2005, pp. 507-540.

[58] Colombo, A., Manzinali, G., Ghidoni, A., Noventa, G., Franciolini, M., Crivellini, A., and Bassi, F., "A p-adaptive implicit discontinuous Galerkin method for the under-resolved simulation of compressible turbulent flows," 7nd European Conference on Computational Fluid Dynamics, 2018.

[59] Naddei, F., de la Llave Plata, M., Couaillier, V., and Coquel, F., "A comparison of refinement indicators for p-adaptive simulations of steady and unsteady flows using discontinuous Galerkin methods," Journal of Computational Physics, Vol. 376 , 2019, pp. 508-533.

[60] Sen, S., Mittal, S., and Biswas, G., "Flow past a square cylinder at low Reynolds numbers," International Journal for Numerical Methods in Fluids, Vol. 67, 2011, pp. 1160 - 1174. https://doi.org/10.1002/fld.2416

[61] Li, Z., "Analysis of 2D unsteady flow past a square cylinder at low Reynolds numbers with CFD and a mesh refinement method," WSEAS Trans. on Fluid Mech, Vol. 12, 2017.

[62] Chalmers, N., Agbaglah, G., Chrust, M., and Mavriplis, C., "A parallel hp-adaptive high order discontinuous Galerkin method for the incompressible Navier-Stokes equations," Journal of Computational Physics: X, Vol. 2, 2019, p. 100023.

[63] Hoffman, J., "Computation of mean drag for bluff body problems using Adaptive DNS/LES," SIAM J. Scientific Computing, Vol. 27, 2005, pp. 184-207. https://doi.org/10.1137/040614463.

[64] Meliga, P., Boujo, E., Pujals, G., and Gallaire, F., "Sensitivity of aerodynamic forces in laminar and turbulent flow past a square cylinder," Physics of Fluids, Vol. 26, No. 10, 2014, p. 104101.

[65] Leicht, T., and Hartmann, R., "Anisotropic mesh refinement for discontinuous Galerkin methods in two-dimensional aerodynamic flow simulations," Int. J. Numer. Meth. Fluids, Vol. 56, 2008, pp. 2111-2138. https://doi.org/10.1002/fld.1608 
[66] Pagnutti, D., and Ollivier-Gooch, C., "A generalized framework for high order anisotropic mesh adaptation," Computers \& structures, Vol. 87, No. 11-12, 2009, pp. 670-679.

[67] Yano, M., and Darmofal, D. L., "Case C1. 3: Flow over the NACA 0012 airfoil: Subsonic inviscid, transonic inviscid, and subsonic laminar flows," First international workshop on high-order CFD methods, 2012.

[68] Balan, A., Woopen, M., and May, G., "Adjoint-based hp-adaptation for a class of high-order hybridized finite element schemes for compressible flows," 21st AIAA Computational Fluid Dynamics Conference, 2013, p. 2938.

[69] Swanson, R. C., and Langer, S., "Comparison of NACA 0012 laminar flow solutions: structured and unstructured grid methods," 2016.

[70] Sari, J., Cremonesi, F., Khalloufi, M., Cauneau, F., Meliga, P., Mesri, Y., and Hachem, E., "Anisotropic adaptive stabilized finite element solver for RANS models," International Journal for Numerical Methods in Fluids, Vol. 86, No. 11, 2018, pp. 717-736.

[71] Baranyi, L., and Lakatos, K., "Computational fluid dynamics analysis of low Reynolds number flow around stationary and oscillating cylinders,” 2004.

[72] Norberg, C., "An experimental investigation of the flow around a circular cylinder: influence of aspect ratio," Journal of Fluid Mechanics, Vol. 258, 1994, pp. 287-316. 